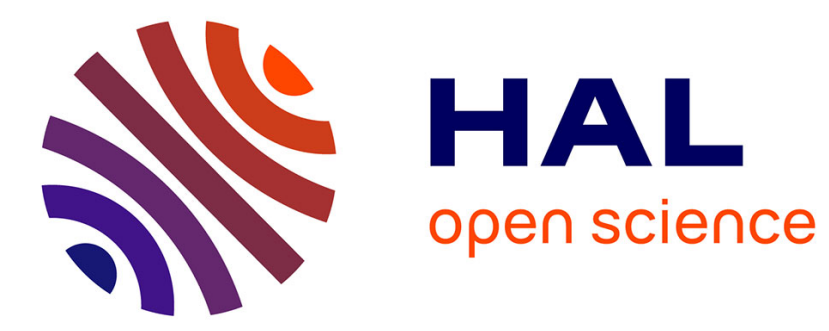

\title{
The diffusion of economic activity across space: a new approach
}

Carmen Camacho, Agustín Pérez-Barahona

\section{To cite this version:}

Carmen Camacho, Agustín Pérez-Barahona. The diffusion of economic activity across space: a new approach. 2017. halshs-01670532

\section{HAL Id: halshs-01670532 \\ https://shs.hal.science/halshs-01670532}

Preprint submitted on 21 Dec 2017

HAL is a multi-disciplinary open access archive for the deposit and dissemination of scientific research documents, whether they are published or not. The documents may come from teaching and research institutions in France or abroad, or from public or private research centers.
L'archive ouverte pluridisciplinaire HAL, est destinée au dépôt et à la diffusion de documents scientifiques de niveau recherche, publiés ou non, émanant des établissements d'enseignement et de recherche français ou étrangers, des laboratoires publics ou privés. 


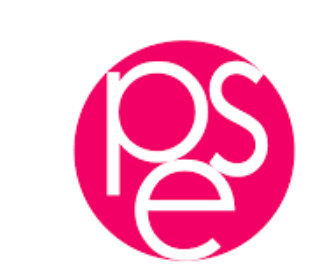

PARISSCHOOLOFECONOMICS
ECOLED'ECONOMIEDEPARIS

WORKING PAPER Nº $2017-61$

The diffusion of economic activity across space: a new approach

Carmen Camacho

Agustin Perez-Barahona

JEL Codes: C62, 04, R11

Keywords: Control, Spatial dynamics, Ramsey model, Partial differential equations

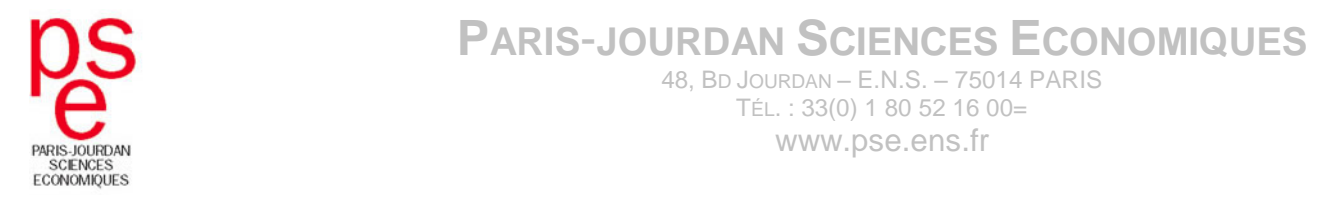

CEnTRE NATIONAL DE LA RECHERCHE SCIENTIFIQUE - ECOLE DES HAUTES ETUdES EN SCIENCES SOCIALES 


\title{
The diffusion of economic activity across space: a new approach
}

\author{
Carmen Camacho \\ Paris School of Economics and CNRS (France) \\ Agustín Pérez-Barahona \\ THEMA, University of Cergy-Pontoise and Ecole Polytechnique (France*)
}

December 1, 2017

\begin{abstract}
Dynamic spatial theory has been a fruitful approach to understand economic phenomena involving time and space. However, this new field has opened a set of questions still unresolved in the literature. For instance, the identification of the social optimal allocation of economic activity across time and space has not been ensured yet in economic growth. By means of a monotone method, we study in this paper the optimal solution of spatial Ramsey-type models. We analytically prove, under fairly general assumptions, the existence of a unique social optimum. The iterative nature of this approach also allows us to present a new algorithm to simulate the optimal trajectories of the economy. We provide two economic illustrations of our method. Firstly, we apply our existence result to the spatial growth model and to a framework for optimal land-use planning, concluding that these problems are well-posed. We then consider the spatial growth model in order to investigate the importance of capital mobility in economic growth. We particularly underline the spatial dynamic implications of this feature on social welfare and income inequality.
\end{abstract}

Keywords: Control, Spatial dynamics, Ramsey model, Partial differential equations.

Journal of Economic Literature: C62, O4, R11.

${ }^{*}$ Corresponding author at: THEMA, U. of Cergy-Pontoise, 33 Boulevard du Port, 95011 Cergy-Pontoise cedex France; e-mail: agustin.perez-barahona@u-cergy.fr. 


\section{Introduction}

A recent strand of the literature on spatial economics has introduced diffusion mechanisms in order to understand the allocation of economic activity across time and space. This new approach turned out to be useful for explaining a set of spatial-dynamic phenomena, such as the mobility of production factors (Boucekkine et al., 2013a; and Fabbri, 2016), the dissemination of technology (Desmet and Rossi-Hansberg, 2009, 2014 and 2015), the spatial allocation of natural resources (Brock and Xepapadeas, 2008), or the spread of pollution due to the economic activity (Camacho and Perez-Barahona, 2015). However, this framework has come across serious methodological issues, without conclusive answers from either the economic or the mathematical literature. The aim of our paper is to contribute in this regard, providing a general analytical tool to study the solution of spatial Ramsey-type models.

Spatial Ramsey-type models are based on the spatial generalization of the RamseyCass-Koopmans model, first presented in Brito (2004) and Boucekkine et al. (2009). This framework considers a forward-looking decision maker that chooses the optimal levels of control variables for a given spatial region and time span. In this problem the social planner also takes into account how the state variables geographically spread. The local levels of consumption are the usual example of controls, with capital or pollution as state variables. This is, indeed, an optimal control problem where the law of motion of the state variables is represented by means of partial differential equations (hereafter PDE). In these PDE the partial derivatives, with respect to space and to time, describe the spatio-temporal progress of the corresponding state variable.

The benchmark for this type of set-up is the spatial growth model, which adds spatial structure to the standard Ramsey model. In this framework the policy maker takes into account the welfare or utility of all the individuals distributed in space, considered over a certain period of time. Moreover capital is assumed to be mobile across locations, where the corresponding spatio-temporal law of motion is a non-linear parabolic PDE. Considering this approach, Boucekkine et al. (2013a) and Fabbri (2016) point out the role played by space in economic growth, which turns out to significantly affect the dynamical properties of the economy. Boucekkine et al. (2013a) specifically show that transitional dynamics can arise, despite of constant returns to capital, in the AK growth model when we incorporate the spatial structure. In environmental economics, Camacho and Perez-Barahona (2015) provide another example of spatial Ramsey-type models. This paper studies the optimal use of land, encompassing land 
use activities (e.g., production, housing or pollution abatement) and environmental degradation. They focus on the spatial externalities of land use management as drivers of spatial patterns: land is immobile by nature, but local actions affect the whole space since pollution flows across locations resulting in environmental damages. This stylized model manages to reproduce a great variety of spatial patterns related to the interaction between land use activities and the environment. In particular, in line with Costello and Polasky (2008), they show that the spatial connectivity (due to the dispersal process), together with the particular characteristics of each location, can economically rationalize the creation of (temporal or permanent) natural reserves. ${ }^{1}$

From a general perspective, spatial Ramsey-type models have been useful to describe the dynamic interaction of economic agents that are spatially distributed. Nevertheless, this framework has opened serious methodological concerns. Within the context of social interactions, Mossay and Picard (2015) and Blanchet et al. (2016) notice important difficulties in ensuring the existence and uniqueness of equilibrium when we incorporate space into the problem. Considering the spatial Ramsey-type framework, papers such as Boucekkine et al. (2009), Boucekkine et al. (2013a,b) and Camacho and Perez-Barahona (2015) identified this trouble too, when one includes both time and space dimensions: although a set of necessary conditions can be provided for a general problem, neither the existence nor the uniqueness of solution has been proven so far. ${ }^{2}$

In this paper we generalize the spatial Ramsey set-up. We then introduce a monotone method, based on Pao (1992), in order to analyze the solution of this type of models. Under fairly general assumptions our method allows us to prove the existence of the unique optimal solution when the time horizon is finite. To the best of our knowledge this is the first paper dealing with the existence and uniqueness of the solution to an optimal control problem governed by a non-linear parabolic PDE. Moreover, the iterative nature of this approach also allows us to present a new algorithm to find numerically the optimal trajectories of the economy. We underline the value of our method with two economic illustrations. One is analytical, the other is numerical.

\footnotetext{
${ }^{1}$ Natural reserves are typically justified under the presence of biological objectives such as biodiversity conservation. On economic grounds, however, that is not the case without explicitly including the spatial dimension.

${ }^{2}$ We can then say that the problem is ill-posed in the sense of Hadamard (1923). Not being able to ensure the existence of solution is obviously problematic. The non-uniqueness concern is not less important because it frequently implies indeterminacy of equilibrium in dynamic contexts. In this regard, many economists consider that models with indeterminacy are inappropriate for economic analysis (among others, Kehoe et al., 1990; and Acemoglu, 2009).
} 
We first study the existence and uniqueness of social optimum in the spatial growth model. We consider as well the optimal land-use framework of Camacho and PérezBarahona (2015) for the spread of local pollutants. From the analytical perspective, the applicability of our method mainly reduces to ensure the existence of upper and lower solutions. These are a sort of boundaries to the Pontryagin conditions that characterize the social optimum. After identifying these boundaries, we conclude that the solution of these two models exists and is unique. Consequently, these problems can be considered as well-posed. This property is key to be able to provide appropriate tools for policymaking, and in particular for the implementation of the social optimum, that involves time and spatial dimensions.

Regarding to the numerical application, we investigate the importance of capital mobility in economic growth. In order to address this question we use the spatial growth model, where capital can flow across locations and agents are continuously distributed in the space. ${ }^{3}$ Barro et al. (1995) present an open-economy neoclassical growth model, without explicitly including spatial structure. Under perfect capital mobility, which is defined as the absence of institutional barriers to spatial capital flows, they observe that transitional dynamics only occurs if capital is irreversible together with the presence of adjustment costs (see also Barro and Sala-i-Martin, 2004). We find in our paper that, for plausible values of the parameters, geography can be naturally considered as an adjustment cost for capital. More specifically, the spatial structure, which is explicitly represented in our set up by the diffusion process, allows the neoclassical growth model with perfect capital mobility and irreversibility to avoid immediate adjustment. In terms of social welfare the model shows that capital mobility is beneficial. When capital is mobile the economy manages to reduce the negative effect of diminishing returns to capital. Capital moves to locations where it is less abundant (and, thus, with higher shadow price). Therefore, the global impact of decreasing returns to scale reduces. Finally, our method also allows us to study the effect of capital mobility on the evolution of (spatial) income inequality. We assume that locations are spatially heterogenous with respect to their initial levels of capital and, consequently, income inequality arises. The simulations show that capital mobility significantly reduces the Gini coefficient of the economy at any time. In fact, improving capital mobility reduces the importance of the initial conditions because capital diffuses to locations with lower initial endowment. This conclusion is in line with the observations made by Galor (1996) or Quah (1996a,b).

\footnotetext{
${ }^{3}$ For the importance of considering a continuum of locations in spatial economics see, for instance, Desmet and Rossi-Hansberg (2009 and 2010) and Thisse (2010).
} 
The paper is structured as follows. Section 2 describes a general spatial version of the Ramsey model. Section 3 presents the result of existence and uniqueness of social optimum. In Section 4 we apply our approach to the two examples of spatial Ramsey-type models, introducing as well the numerical method. Finally, we present our conclusions in Section 5.

\section{The general problem}

The objective of the policy maker is to maximize the aggregated social welfare in a bounded region of space $\Omega \subset \mathbb{R}$ over a finite time period $[0, T]$. To do so, she chooses a time trajectory for a variable $c$ in each location, taking into account the dynamics of a state variable $u_{1}$. The choice of the control $c$ affects in turn the dynamics of the state. More precisely, the policy maker maximizes the welfare provided by $c$, aggregating felicity over time and space plus a measure the final spatial situation of the economy, which is represented by a scrap value function $\Psi$, as follows:

$$
\max _{c} \int_{0}^{T} \int_{\Omega} U(c(x, t)) g(x, t) d x d t+\int_{\Omega} \Psi\left(u_{1}(x, T), x\right) d x
$$

subject to:

$$
\left\{\begin{array}{l}
u_{1, t}(x, t)-u_{1, x x}(x, t)=f_{1}\left(u_{1}, c\right), \\
\lim _{x \rightarrow \delta \Omega} u_{1, x}(x, t)=0, \\
u_{1}(x, 0)=u_{1,0}(x) \geq 0 \text { given. }
\end{array}\right.
$$

We assume in the objective function (1) that $U \in C^{2}\left(\mathbb{R}^{+}\right)$is measurable, increasing and concave. As observed in the introduction, the level of local consumption $c(x, t)$ is the typical example of control variable in this type of models. The function $g(x, t)$ represents the usual spatial and time discounting. For instance, following Boucekkine et al. (2013b), one can consider the example $g(x, t)=\xi(x) e^{-\rho t}$. This functional form assumes that time is exponentially discounted as in the standard growth model, where $\rho>0$ is the time-discount rate. Moreover, function $\xi(x)$ is assumed to "rapidly decrease" in order to discount space. ${ }^{4}$ As in Camacho and Pérez-Barahona (2015), the spatial discounting can represent the importance that the policy maker gives to each location of

\footnotetext{
${ }^{4}$ Notice that both time and space discounting can be dropped in our set-up since time period and space are assumed to be bounded. Section 5 provides further remarks about the decision horizon.
} 
the economy. Moreover, the scrap function can also include space and time discounting since the convergence of the corresponding integral term is required as well. We additionally assume that $\Psi \in C^{1}\left(D\left(u_{1}\right), \Omega\right)$, defined on the domain of $u_{1}$, is a positive function with bounded derivative $\Psi_{1}^{\prime}>0$.

The first equation in (2) describes the spatial and time dynamics of the state variable $u_{1}$. This is a parabolic PDE, where $u_{1, t}$ denotes the partial derivative with respect to time and $u_{1, x x}$ is the second partial derivative with respect to space. As pointed out in the introduction, this expression can represent the spatio-temporal spread of capital in an economic growth framework. Identifying $u_{1}(x, t)$ with the capital of location $x$ at time $t, k(x, t)$, the law of motion of capital can be written as

$$
k_{t}(x, t)-k_{x x}(x, t)=I(x, t),
$$

where $I(x, t)$ represents the net investment of a location $x$ at time $t$. The main difference with respect to the standard law of motion without space is the term $-k_{x x}(x, t)$. As in Boucekkine et al. (2009) and Boucekkine et al. (2013a), it reflects that capital flows from regions with lower marginal productivity of capital (i.e., with abundant capital) to the higher ones, with relatively less capital. They actually show that equation (3) can be economically justified as a balance trade equilibrium in a bounded region.

Camacho and Pérez-Barahona (2015) provide another example of this type of equation in a land-use framework, where the dynamics of pollution $p(x, t)$ follows

$$
p_{t}(x, t)-p_{x x}(x, t)=E(x, t)
$$

$E(x, t)$ summarizes the emissions in time $t$ of a single source located at $x$. The term $-p_{x x}(x, t)$ above states that a pollutant diffuses from regions where its concentration is high to regions of lower concentration (Fick's law of diffusion). Indeed, equation (4) is the well-known model in physics called the Gaussian plume, which is frequently used to describe the spread of pollution across locations.

The second expression in (2) is known in the literature as the Neumann boundary condition. It characterizes the behaviour of $u_{1}$ in the spatial frontier, assuming that there is no flow of the state variable (capital, for instance) at locations that are far away from the origin. ${ }^{5}$ Finally, the last equation is the initial spatial distribution of $u_{1}$, which is assumed to be a known positive function $u_{1,0}(x) \in C(\bar{\Omega})$.

\footnotetext{
${ }^{5}$ This condition can be relaxed by assuming a circular space, so $k(0, t)=k(2 \pi, t)$. For further discussion see Boucekkine et al. (2013a,b).
} 
In the optimal control literature few papers deal with the existence of unique solution in this type of problems. Unique solution exists in some particular cases, namely a linear $f_{1}\left(u_{1}, c\right)$ (Lions, 1966a,b; and Barbu and Precupanu, 2012) or under specific non-linearities with very limited interaction between the state and the control variables (Lions, 1966a,b and 1972; and Ahmed, 1977). Still, using an appropriated maximum principle, Pontryagin conditions can be associated to general non-linear problems. Fattorini (1990) and Raymond and Zidani (1999) prove that the set of Pontryagin conditions is necessary and sufficient to characterize the solutions of this problem. However, to the best of our knowledge, we have not found any existence result for this type of dynamical systems.

Using the Ekeland (1974) variational principle, we obtain the associated set of Pontryagin necessary conditions as: ${ }^{6}$

$$
(O)\left\{\begin{array}{l}
u_{1, t}(x, t)-u_{1, x x}(x, t)=f_{1}\left(u_{1}, c\right), \\
u_{2, t}(x, t)+u_{2, x x}(x, t)=f_{2}\left(u_{1}, u_{2}, c\right), \\
u_{2} \frac{\partial f_{1}}{\partial c}=-U^{\prime}(c) g(x, t), \\
\lim _{x \rightarrow \delta \Omega} u_{i, x}(x, t)=0, \quad i=1,2 \\
u_{1}(x, 0)=u_{1,0}(x) \geq 0, \text { given, } \\
u_{2}(x, T)=\Psi_{1}^{\prime}\left(u_{1}(x, T), x\right)
\end{array}\right.
$$

where $\delta \Omega=\bar{\Omega} \backslash \Omega$ is $\Omega$ 's frontier and $u_{2}$ is the corresponding co-state variable (shadow price). As we will see in the next section, ensuring the existence of unique solution to the system $(O)$ is far from being obvious due to presence of two coupled non-linear parabolic PDE, where one of them is time reversed.

Let us also observe that in the literature of spatial economics there are some pragmatical, but constrained, ways to tackle the problem. A linear utility in the objective function allows Boucekkine et al. (2009) to decouple the Pontryagin conditions, solving each block of PDE separately. Using dynamic programming in Hilbert spaces, Boucekkine et al. (2013a) overcome the unicity problem in a spatial version of the AK growth model, where space is a circle and the time horizon is infinite. Finally, other papers focus on particular solutions (spatially periodic or time-constant solutions) to the spatial Ramsey-type model as Brito (2004) or Brock and Xepapadeas (2008).

\footnotetext{
${ }^{6}$ See Appendix A for details.
} 


\section{Existence and uniqueness of the solution}

We prove in this section the existence of the unique solution to our system of non-linear PDE of real functions on $\Omega \times[0, T]$. For the ease of presentation, since $c$ can be expressed as a function of $u_{1}$ and $u_{2}$, let us rewrite $(O)$ as:

$$
(I)\left\{\begin{array}{l}
u_{1, t}(x, t)-u_{1, x x}(x, t)=f_{1}\left(u_{1}, u_{2}\right), \\
u_{2, t}(x, t)+u_{2, x x}(x, t)=f_{2}\left(u_{1}, u_{2}\right), \\
\lim _{x \rightarrow \delta \Omega} u_{i, x}(x, t)=0, \quad i=1,2 \\
u_{1}(x, 0)=u_{1,0}(x) \geq 0, \text { given, } \\
u_{2}(x, T)=\Psi_{1}^{\prime}\left(u_{1}(x, T), x\right)
\end{array}\right.
$$

This system is a generalization of the set of necessary and sufficient conditions identified in Boucekkine et al. (2009). One should observe that $(I)$ is composed of two PDE. The one corresponding to $u_{1}$ is parabolic. However, as we will show later, the second PDE is parabolic only after reversing time. The system includes as well conditions on the partial derivatives of $u_{1}$ and $u_{2}$ at the spatial borders, together with the given initial distribution for $u_{1}$. Moreover $\Psi_{1}^{\prime}\left(u_{1}(x, T), x\right)$ determines the final distribution for $u_{2}$, which is a function of the final distribution for $u_{1}$. This last condition ties the final distribution of the state variable to the final distribution of the associated co-state. As in Camacho et al. (2008) it reveals that, at the end of the planning horizon, the shadow price of the state variable equals the marginal social scrap value of the stock.

In this paper we introduce a monotone method based on Pao (1992). These methods were widely used to prove the existence of solution for systems of PDE, of parabolic and hyperbolic type, with both boundary and initial conditions (see, among others, Sattinger, 1972; Changdra et al., 1978; and Bebernes and Schimitt, 1979). Before going into the details of the proof, let us briefly describe the idea of our method. In a nutshell, we build two converging sequences of functions that unambiguously encircle the unique solution to the system. First, we define and identify appropriate upper and lower solutions of the original problem $(I)$, which are typically easier to ensure than the solution itself. Starting then from these solutions, we describe sequences of associated linear problems, which have unique solution. We finally show that these associated problems converge to the original one $(I)$ from above (below), starting with the upper (lower) solution.

More formally, let us begin by describing the norms that we will use to size functions 
and variables in our method. We consider two norms along the proof, the usual norm in $\mathbb{R}^{2}$ and the supremum norm. For every $u(x, t) \equiv\left(u_{1}(x, t), u_{2}(x, t)\right) \in \mathbb{R}^{2}$ and at every $(x, t) \in \Omega \times[0, T]$, the Euclidean norm in $\mathbb{R}^{2}$ is defined as

$$
\|u(x, t)\| \equiv\left|u_{1}(x, t)\right|+\left|u_{2}(x, t)\right| .
$$

We also make use of the supremum norm $\|\cdot\|_{0}$, defined for any $u \in \mathbb{R}^{2}$ :

$$
\|u\|_{0} \equiv \sup _{(x, t) \in \Omega \times[0, T]}\|u\|
$$

As pointed out above, the dynamics of $u_{2}$ is described by a reversed-time parabolic PDE. Consequently, one of the key ingredients of the proof is to reverse time in the second $\mathrm{PDE}$ in $(I)$. We define in this regard the variables $w_{1}$ and $w_{2}$ as

$$
\begin{aligned}
& w_{1}(x, t) \equiv u_{1}(x, T-t), \\
& w_{2}(x, t) \equiv u_{2}(x, T-t) .
\end{aligned}
$$

Then, the dynamics of $w_{2}$ is described by a parabolic PDE with a known initial distribution $\Psi_{1}^{\prime}\left(w_{1}(x, 0), x\right)$ :

$$
\left\{\begin{array}{l}
w_{2, t}(x, t)-w_{2, x x}(x, t)=-f_{2}\left(w_{1}, w_{2}\right), \\
w_{2}(x, 0)=\Psi_{1}^{\prime}\left(w_{1}(x, 0), x\right) .
\end{array}\right.
$$

The system $(I)$ actually mixes two types of non-linear parabolic PDE, where one is parabolic only after reversing time (see 5 above). We aim then at extending the monotone method of Pao (1992), considering in our paper systems of mixed types of parabolic PDE. In order to do so, we need to define adequate upper and lower solutions. We say that two functions $\hat{u}=\left(\hat{u}_{1}, \hat{u}_{2}\right)$ and $\tilde{u}=\left(\tilde{u}_{1}, \tilde{u}_{2}\right)$ are upper and lower solutions to $(I)$ if they satisfy for every $t$ :

$$
\begin{aligned}
& \tilde{u}_{1, t}(x, t)-\tilde{u}_{1, x x}(x, t)-f_{1}\left(\tilde{u}_{1}, \tilde{u}_{2}\right) \leq 0 \leq \hat{u}_{1, t}(x, t)-\hat{u}_{1, x x}(x, t)-f_{1}\left(\hat{u}_{1}, \hat{u}_{2}\right), \\
& \hat{u}_{2, t}(x, t)+\hat{u}_{2, x x}(x, t)-f_{2}\left(\hat{u}_{1}, \hat{u}_{2}\right) \leq 0 \leq \tilde{u}_{2, t}(x, t)+\tilde{u}_{2, x x}(x, t)-f_{2}\left(\tilde{u}_{1}, \tilde{u}_{2}\right), \\
& \lim _{x \rightarrow \delta \Omega} \hat{u}_{i, x}(x, t)=0, \lim _{x \rightarrow \delta \Omega} \tilde{u}_{i, x}(x, t)=0, i=1,2,
\end{aligned}
$$

with $\tilde{u}_{1} \leq \hat{u}_{1}, \tilde{u}_{2} \leq \hat{u}_{2}$, and

$$
\begin{aligned}
& \tilde{u}_{1}(x, 0)<u_{1}(x, 0)<\hat{u}_{1}(x, 0), \\
& \tilde{u}_{2}(x, 0)<u_{2}(x, 0)<\hat{u}_{2}(x, 0),
\end{aligned}
$$


for all $x \in \Omega .^{7}$

Notice that due to the presence of a reversed-time parabolic PDE, considering (5) the PDE condition for $\hat{u}_{2}$ and $\tilde{u}_{2}$ in (6) becomes

$$
\tilde{w}_{2, t}(x, t)-\tilde{w}_{2, x x}(x, t)+f_{2}\left(\tilde{w}_{1}, \tilde{w}_{2}\right) \leq 0 \leq \hat{w}_{2, t}(x, t)-\hat{w}_{2, x x}(x, t)+f_{2}\left(\hat{w}_{1}, \hat{w}_{2}\right) .
$$

That is to say, $\hat{w}_{2}$ and $\tilde{w}_{2}$ are, respectively, the upper and lower solution of the corresponding parabolic PDE, as it is usually defined in the literature. Moreover,

$$
\tilde{w}_{2}(x, 0)<\Psi_{1}^{\prime}\left(u_{1}(x, T), x\right)<\hat{w}_{2}(x, 0) .
$$

For $i=1,2, \tilde{u}_{i}, \hat{u}_{i}$ are required to be $C(\bar{\Omega} \times[0, T]) \cap C^{1,2}(\Omega \times[0, T])$, that is, the are continuous in $\bar{\Omega} \times[0, T]$, continuously differentiable in $t$ and twice continuously differentiable in $x$ for all $(x, t) \in \Omega \times[0, T]$.

Having established the appropriate definition of lower and upper solutions, let us also define the set of continuous functions between $\tilde{u}$ and $\hat{u}$ as

$$
<\tilde{u}, \hat{u}>\equiv\left\{\left(u_{1}, u_{2}\right) \in C(\Omega \times[0, T]):\left(\tilde{u}_{1}, \tilde{u}_{2}\right) \leq\left(u_{1}, u_{2}\right) \leq\left(\hat{u}_{1}, \hat{u}_{2}\right)\right\}
$$

The interest of $\langle\tilde{u}, \hat{u}\rangle$ is that it defines the precise space where solutions will be confined, i.e., all solutions are limited from below by $\tilde{u}$ and from above by $\hat{u}$ (see Theorem 2 at the end of this section).

As usual in this literature, we need to impose boundedness conditions on initial distributions and functions $f_{1}$ and $f_{2}$ in order to avoid explosive solutions:

Assumption 1. $u_{1,0}(x) \in C(\bar{\Omega})$ is measurable. The initial distributions verify that

$$
\left\{\begin{array} { l } 
{ \tilde { u } _ { 1 } ( x , 0 ) < h _ { 1 } e ^ { h _ { 2 } | x | ^ { 2 } } } \\
{ u _ { 1 } ( x , 0 ) < h _ { 1 } e ^ { h _ { 2 } | x | ^ { 2 } } } \\
{ \hat { u } _ { 1 } ( x , 0 ) < h _ { 1 } e ^ { h _ { 2 } | x | ^ { 2 } } }
\end{array} \quad \text { and } \quad \left\{\begin{array}{l}
\tilde{u}_{2}(x, 0)<h_{1} e^{h_{2}|x|^{2}} \\
\hat{u}_{2}(x, 0)<h_{1} e^{h_{2}|x|^{2}}
\end{array}\right.\right.
$$

for some positive constants $h_{1}, h_{2}$ with $h_{2}<\frac{1}{4 T}$.

Assumption 2. There exist bounded functions $\underline{a}_{i}=\underline{a}_{i}(x, t), \bar{a}_{i}=\bar{a}_{i}(x, t), \underline{c}_{i}=\underline{c}_{i}(x, t)$ and $\bar{c}_{i}=\bar{c}_{i}(x, t)$, with $i=1,2$, such that for every $u, v \in<\tilde{u}, \hat{u}>$, where $u=\left(u_{1}, u_{2}\right)$ and $v=\left(v_{1}, v_{2}\right),\left(f_{1}, f_{2}\right)$ satisfies

$$
-\underline{a}_{1}\left(u_{1}-v_{1}\right)-\underline{a}_{2}\left(u_{2}-v_{2}\right) \leq f_{1}\left(u_{1}, u_{2}\right)-f_{1}\left(v_{1}, v_{2}\right) \leq \bar{a}_{1}\left(u_{1}-v_{1}\right)+\bar{a}_{2}\left(u_{2}-v_{2}\right),
$$

\footnotetext{
${ }^{7}$ We say that a function $m$ is larger than another function $m^{\prime}$ in $\Omega \times[0, T]$, i.e., $m \geq m^{\prime}$, if and only if $m(x, t) \geq m^{\prime}(x, t)$ for all $(x, t) \in \Omega \times[0, T]$.
} 


$$
-\underline{c}_{1}\left(u_{1}-v_{1}\right)-\underline{c}_{2}\left(u_{2}-v_{2}\right) \leq f_{2}\left(u_{1}, u_{2}\right)-f_{2}\left(v_{1}, v_{2}\right) \leq \bar{c}_{1}\left(u_{1}-v_{1}\right)+\bar{c}_{2}\left(u_{2}-v_{2}\right),
$$

for $u_{1} \geq v_{1}$ and $u_{2} \geq v_{2}$. Furthermore, functions $\underline{a}_{1}$ and $\bar{c}_{2}$ are Hölder continuous in $\Omega \times[0, T]$.

We restrict $f_{i}$ domain to sets in which Assumption 3 below is verified. We denote these reduced domains for $f_{1}$ and $f_{2}$ by $J_{1}$ and $J_{2}$, respectively.

Assumption 3. For every $u, v \in<\tilde{u}, \hat{u}>$, either $f_{1}$ is increasing in both $u_{1}$ and $u_{2}$ and $\underline{a}_{1} \geq 0$, or $\underline{a}_{2} \leq 0$. Similarly, either $f_{2}$ is decreasing in both $u_{1}$ and $u_{2}$ and $\bar{c}_{2} \geq 0$, or $\bar{c}_{1} \leq 0$.

Finally, we include some continuity assumptions on functions $f_{i}$ :

Assumption 4. For $i=1,2$, functions $f_{i}$ are Hölder continuous in $J_{1} \times J_{2}$ for some subset $J_{1} \times J_{2} \subseteq \mathbb{R}^{2}$.

Defining functions $M_{1}$ and $M_{2}$ as

$$
\begin{aligned}
& M_{1} \equiv \max \left\{\left|\underline{a}_{1}\right|,\left|\underline{a}_{2}\right|,\left|\bar{a}_{1}\right|,\left|\bar{a}_{2}\right|\right\}, \\
& M_{2} \equiv \max \left\{\left|\underline{c}_{1}\right|,\left|\underline{c}_{2}\right|,\left|\bar{c}_{1}\right|,\left|\bar{c}_{2}\right|\right\}
\end{aligned}
$$

functions $f_{1}$ and $f_{2}$ are Lipschitz continuous since $M_{i}=M_{i}(x, t)$ are bounded in $\Omega \times$ $[0, T]$. That is to say, there exist bounded functions $M_{i}=M_{i}(x, t)$ in $\Omega \times[0, T]$ such that

$$
\left|f_{i}\left(u_{1}, u_{2}\right)-f_{i}\left(v_{1}, v_{2}\right)\right| \leq M_{i}|u-v|
$$

for any $u, v$ in $<\tilde{u}, \hat{u}>$.

With functions $\underline{a}_{1}$ and $\bar{c}_{2}$ from Assumption 2, we build functions $F_{1}$ and $F_{2}$ as

$$
\begin{aligned}
& F_{1}\left(x, t, u_{1}, u_{2}\right) \equiv f_{1}\left(u_{1}, u_{2}\right)+\underline{a}_{1}(x, t) u_{1}, \\
& F_{2}\left(x, t, u_{1}, u_{2}\right) \equiv f_{2}\left(u_{1}, u_{2}\right)-\bar{c}_{2}(x, t) u_{2} .
\end{aligned}
$$

By Assumptions 2 and 4, functions $F_{i}$ are Hölder continuous in $\Omega \times[0, T] \times<\tilde{u}, \hat{u}>$, for $i=1,2$. Furthermore, we also define two linear operators of $u_{1}$ and $u_{2}, \mathbb{L}_{1}$ and $\mathbb{L}_{2}$, as

$$
\begin{aligned}
& \mathbb{L}_{1} u_{1} \equiv u_{1, t}-u_{1, x x}+\underline{a}_{1} u_{1}, \\
& \mathbb{L}_{2} u_{2} \equiv u_{2, t}+u_{2, x x}-\bar{c}_{2} u_{2} .
\end{aligned}
$$

\footnotetext{
${ }^{8}$ Our results can be easily extended to functions $f_{i}$ that depend on $x$ and $t$, i.e., $f_{i}\left(x, t, u_{1}, u_{2}\right)$. In this case, $f_{i}$ would be Hölder continuous in $\Omega \times[0, T] \times J_{1} \times J_{2}$.
} 
Introducing the functions $F_{i}$ and the operators $\mathbb{L}_{i}$ defined above, we can rewrite the two $\mathrm{PDE}$ in $(I)$ as

$$
\mathbb{L}_{i} u_{i}=F_{i}\left(u_{1}, u_{2}\right)
$$

in $\Omega \times[0, T]$ for $i=1,2$.

As pointed out before, the PDE corresponding to $u_{2}$ is parabolic after reversing time. Therefore, we define an alternative operator to $\mathbb{L}_{2}$ in order to consider only parabolic PDEs:

$$
\mathbb{L}_{2}^{\prime} w_{2} \equiv w_{2, t}-w_{2, x x}+\bar{c}_{2} w_{2} .
$$

Taking then the operator $\mathbb{L}_{2}^{\prime}$, the PDE in (5) becomes

$$
\mathbb{L}_{2}^{\prime} w_{2}=-F_{2}\left(w_{1}, w_{2}\right)
$$

This new writing turns out to be extremely useful in the upcoming Theorem 1. It allows us to consider sequences of single linear parabolic PDE that we will use in order to prove the existence of the unique solution for $(I)$. As we briefly explained before, this theorem begins by building two monotonic sequences of functions. We set up, on the one hand, a non-increasing sequence of continuous functions of time and space $\left\{\bar{u}_{1}^{k}, \bar{u}_{2}^{k}\right\}_{k \in \mathbb{N}}$ and, on the other hand, a non-decreasing sequence of continuous functions $\left\{\underline{u}_{1}^{k}, \underline{u}_{2}^{k}\right\}_{k \in \mathbb{N}}$. Without loss of generality, we establish $\left\{\bar{u}_{1}^{k}\right\}_{k \in \mathbb{N}}$ initiating the sequence with the upper solution $\left\{\hat{u}_{1}, \hat{u}_{2}\right\}$. At every step, we generate $\bar{u}_{1}^{k}$ taking $\left(\bar{u}_{1}^{k-1}, \bar{u}_{2}^{k-1}\right)$. Then, with $\bar{w}_{1}^{k}(x, t)=\bar{u}_{1}^{k}(x, T-t)$ and $\left(\bar{w}_{1}^{k-1}, \bar{w}_{2}^{k-1}\right)$, we will obtain $\bar{w}_{2}^{k}$. The step ends when we reverse time again to obtain $\bar{u}_{2}^{k}(x, t)=\bar{w}_{2}^{k}(x, T-t)$. We can repeat this procedure at infinitum to build our sequence. Regarding the sequences $\left\{\underline{u}_{1}^{k}\right\}_{k \in \mathbb{N}}$ and $\left\{\underline{u}_{2}^{k}\right\}_{k \in \mathbb{N}}$, we follow a similar strategy, initiating the process with the lower solution $\left\{\tilde{u}_{1}, \tilde{u}_{2}\right\}$. We then prove that these sequences uniformly converge:

$$
\lim _{k \rightarrow \infty} \bar{u}_{i}^{k}=\bar{u}_{i} \text { and } \lim _{k \rightarrow \infty} \underline{u}_{i}^{k}=\underline{u}_{i}, i=1,2 .
$$

Finally we show that $\mathbb{L}^{-1} F$ possesses the contraction property, where we define the diagonal matrix $\mathbb{L} \equiv \operatorname{diag}\left(\mathbb{L}_{1}, \mathbb{L}_{2}\right)$ and the transpose of the vector of $F_{i}$ functions $F \equiv\left(F_{1}, F_{2}\right)^{\prime}$. Hence, the limits of the above sequences coincide and define the unique solution to $(I)$.

Following these steps, Theorem 1 states that problem $(I)$ has a unique solution in $\Omega \times[0, T]$ under the assumptions established before: 
Theorem 1. Under the Assumptions 1-4, the problem

$$
\left\{\begin{array}{l}
u_{1, t}(x, t)-u_{1, x x}(x, t)=f_{1}\left(u_{1}, u_{2}\right), \\
u_{2, t}(x, t)+u_{2, x x}(x, t)=f_{2}\left(u_{1}, u_{2}\right), \\
\lim _{x \rightarrow \delta \Omega} u_{i, x}(x, t)=0, \quad i=1,2 \\
u_{1}(x, 0)=u_{1,0}(x) \text { given, } \\
u_{2}(x, T)=\Psi_{1}^{\prime}\left(u_{1}(x, T), x\right)
\end{array}\right.
$$

has a unique solution $\left(u_{1}, u_{2}\right)$ in $\Omega \times[0, T]$. Moreover, $u=\left(u_{1}, u_{2}\right)$ is the limit of the monotonic sequence $\left\{u_{1}^{k}, u_{2}^{k}\right\}_{k \in \mathbb{N}}$, where $u_{2}^{k}(t, x)=w_{2}^{k}(x, T-t)$. The sequence $\left\{u_{1}^{k}, w_{2}^{k}\right\}_{k \in \mathbb{N}}$ is generated as

$$
\left\{\begin{array}{l}
\mathbb{L}_{1} u_{1}^{k}=F_{1}\left(u_{1}^{k-1}, u_{2}^{k-1}\right), \lim _{x \rightarrow \delta \Omega} u_{1, x}^{k}(x, t)=0, \quad \forall t \geq 0, \\
\mathbb{L}_{2}^{\prime} w_{2}^{k}=-F_{2}\left(w_{1}^{k-1}, w_{2}^{k-1}\right), \lim _{x \rightarrow \delta \Omega} w_{2, x}^{k}(x, t)=0, \quad \forall t \geq 0,
\end{array}\right.
$$

for all $k=1,2, \ldots$ We can choose between two sets of initial conditions and initial distributions. The first set $(\hat{S})$ of conditions below generates a non-increasing sequence, whereas the second set $(\tilde{S})$ generates a non-decreasing sequence:

$$
\begin{aligned}
& (\hat{S})\left\{\begin{array}{l}
\left(u_{1}^{0}, u_{2}^{0}\right)=\left(\hat{u}_{1}, \hat{u}_{2}\right), \\
u_{1}^{k}(x, 0)=\frac{\Upsilon}{k} \hat{u}_{1}(x, 0)+\left(1-\frac{\Upsilon}{k}\right) u_{1}(x, 0), \\
w_{2}^{k}(x, 0)=\frac{\psi}{k} \hat{w}_{2}(x, 0)+\left(1-\frac{\psi}{k}\right) \Psi_{1}^{\prime}\left(w_{1}^{k-1}(x, 0), x\right) ;
\end{array}\right. \\
& (\tilde{S})\left\{\begin{array}{l}
\left(u_{1}^{0}, u_{2}^{0}\right)=\left(\tilde{u}_{1}, \tilde{u}_{2}\right), \\
u_{1}^{k}(x, 0)=\frac{\zeta}{k} \tilde{u}_{1}(x, 0)+\left(1-\frac{\zeta}{k}\right) u_{1}(x, 0), \\
w_{2}^{k}(x, 0)=\frac{\phi}{k} \tilde{w}_{2}(x, 0)+\left(1-\frac{\phi}{k}\right) \Psi_{1}^{\prime}\left(w_{1}^{k-1}(x, 0), x\right),
\end{array}\right.
\end{aligned}
$$

with $0<\zeta<\Upsilon<1$ and $0<\phi<\psi<1$.

Proof. See Appendix B.

We have proven so far that, once a pair of lower and upper solutions is identified, there exists a unique solution $u$ to our original problem between them. A natural question arises, wondering about how many solutions one could find below (above) the specific lower (upper) solution. The answer is that, under the assumptions of the model, we 
will not find any. Therefore, the unique solution of the problem will be unambiguously located between the pair of lower and upper solutions, i.e., $u \in<\tilde{u}, \hat{u}>$. We state this final result in the following theorem:

Theorem 2. Let Assumptions 1-4 hold, and $\tilde{u}$ and $\hat{u}$ be lower and upper solutions to problem $(I)$. Let us further assume that:

i) either $f_{1}$ is increasing in $u_{1}$ and $u_{2}$, with $\underline{a}_{1} \geq 0$; or $\underline{a}_{2} \leq 0$,

ii) either $f_{2}$ is decreasing in $u_{1}$ and $u_{2}$, with $\bar{c}_{2} \geq 0$; or $\bar{c}_{1} \leq 0$.

If $u$ is a solution to $(I)$ then $u \in<\tilde{u}, \hat{u}>$.

Proof. See Appendix C.

\section{Applications}

Let us apply our method to two examples of spatial-Ramsey type models. We consider first the spatial growth model originally introduced by Brito (2004) and Boucekkine et al. (2009). The second example is based on the optimal land-use framework of Camacho and Pérez-Barahona (2015), focusing on the diffusion of local pollutants. In both models ill-posedness had not been solved this far, even under the case of finite decision horizon. Boucekkine et al. (2013a) is an exception in this respect, but they focus on the case of linear production function (AK model). Camacho and Pérez-Barahona (2015) allow for a non-linear production function. However, their analytical results about the existence of unique equilibrium exclusively apply to the time-invariant solution. In our paper we consider instead the whole optimal trajectory.

Making use of Theorems 1 and 2, we will conclude that the social optimum exists and is unique. Hence, when the time horizon is finite, we can confirm that these models are well-posed. From a numerical perspective, we will implement the iterative method presented in Theorem 1 in order to simulate the optimal trajectories of the spatial growth model. In particular, we will apply this algorithm to study the role played by capital mobility in economic growth. ${ }^{9}$

\footnotetext{
${ }^{9}$ Camacho and Pérez-Barahona (2015) already include a numerical analysis of our second example. Their computational setting is alternatively based on a discretization of the Pontryagin conditions, starting from an initial guess for the reversed-time shadow price.
} 


\subsection{The spatial growth model}

Within the general framework of economic growth theory, the spatial Ramsey model considers a policy maker that maximizes utility in a region $\Omega$ of space from time 0 to $T$, subject to the dynamic evolution across time and space of the capital stock. ${ }^{10}$ The corresponding optimal control problem is

$$
\max _{c} \int_{0}^{T} \int_{\Omega} U(c(x, t)) e^{-\rho t} d x d t+\int_{\Omega} \Psi(k(x, T)) e^{-\rho T} d x
$$

subject to

$$
\left\{\begin{array}{l}
k_{t}(x, t)-k_{x x}(x, t)=A f(k(x, t))-\delta k(x, t)-c(x, t), \forall(x, t) \in \Omega \times[0, T] \\
\lim _{x \rightarrow \delta \Omega} k_{x}(x, t)=0 \forall t \in[0, T] \\
k(x, 0)=k_{0}(x)>0 \text { given } \forall x \in \Omega .
\end{array}\right.
$$

The policy maker decides on the consumption $c(x, t)$ of the representative household at every location $x$ and time $t$. In this respect, she takes into account the spatial-dynamic motion of the stock of capital in each location $k(x, t)$. The first constraint in (13) represents the law of motion of $k(x, t)$. The right hand side of this equation is the net investment, where $\delta \in(0,1)$ is the rate of capital depreciation and $f(\cdot)$ is the production technology of the economy, with a scale parameter $A$. As we already observed in Section 2 (see equation 3 ), this expression is a parabolic PDE where $k_{x x}(x, t)$ introduces a new feature with respect to the standard law of motion. This term entirely comes from the spatial mobility of capital, which flows from regions with lower marginal productivity of capital to the higher ones. The second constraint is the usual (Neumann) boundary condition. It assumes that there is no capital flow at locations that are far away from the origin. Finally $k_{0}(x)>0$ is the initial spatial distribution of capital, which is given in the economy.

$\Psi(\cdot)>0$ measures how much the policy maker cares about the final state of capital, or scrap value. It is a continuously differentiable function with bounded and positive derivative. We assume that $U$ and $f$ are both positive, continuous, differentiable and concave functions. Only settings with measurable $k_{0}$ and existing integrals are considered. Following the variational principle introduced in Appendix A, the Pontryagin

\footnotetext{
${ }^{10}$ Spatial economics usually considers space as a physical characteristic of the problem. However, it is possible to think about alternative interpretations such as the idea of "economic distance" suggested by Patel (1964) or Quah (1996a).
} 
optimal conditions associated to this problem are

$$
\left\{\begin{array}{l}
k_{t}(x, t)-k_{x x}(x, t)=A f(k(x, t))-\delta k(x, t)-c(x, t), \forall(x, t) \in \Omega \times[0, T], \\
\lambda_{t}(x, t)+\lambda_{x x}(x, t)=\lambda\left(\delta+\rho-A f^{\prime}(k(x, t))\right), \forall(x, t) \in \Omega \times[0, T], \\
U^{\prime}(c(x, t))=\lambda(x, t), \forall(x, t) \in \Omega \times[0, T] \\
\lim _{x \rightarrow \delta \Omega} k_{x}(x, t)=\lim _{x \rightarrow \delta \Omega} \lambda_{x}(x, t)=0, \forall t \in[0, T], \\
k(x, 0)=k_{0}(x) \text { given, } \forall x \in \Omega, \\
\lambda(x, T)=\Psi^{\prime}(k(x, T)), \forall x \in \Omega,
\end{array}\right.
$$

where $\lambda$ is the corresponding co-state variable, i.e., the shadow price of capital.

\subsubsection{Analytical application}

For this illustration we consider the usual CRRA utility function $U(c)=\frac{c^{1-\sigma}}{1-\sigma}$ with $\sigma>0$, together with a Cobb-Douglas production technology $f(k)=k^{\alpha}$ where $\alpha \in(0,1)$. We assume, for the sake of simplicity, a linear scrapping value $\Psi(k(x, T))=\chi k(x, T)$ with $\chi>0$. Using our general formulation in $(I), f_{1}(k, \lambda)=A f(k(x, t))-\delta k(x, t)-\lambda(x, t)^{-1 / \sigma}$ and $f_{2}(k, \lambda)=\lambda\left(\delta+\rho-A f^{\prime}(k(x, t))\right)$. Let us apply Theorem 1 in order to prove the existence of the unique social optimal solution of the spatial growth model. As we pointed out in the previous section, the main idea of the theorem is to identify upper and lower solutions to the system $(I)$. This will ensure the existence of unique solution within the space that the upper and lower solutions outline. Taking the definition (6)(8) of this type of solutions, one can verify that the following couple is a lower solution to our problem :

$$
(\tilde{k}, \tilde{\lambda})=\left(\left(\frac{\alpha A}{\delta+\rho}\right)^{\frac{1}{1-\alpha}}-\epsilon,\left(A \tilde{k}^{\alpha}-\delta \tilde{k}\right)^{-\sigma}+\epsilon\right)
$$

with $\epsilon>0$ a constant. Moreover, the couple below provides an upper solution:

$$
(\hat{k}, \hat{\lambda})=\left(\varphi e^{\phi t}, \psi e^{\zeta t}\right)
$$

with

$$
\begin{aligned}
& A \alpha \varphi^{\alpha-1} \leq \delta+\rho-\zeta \\
& e^{\frac{\zeta+\phi}{\sigma} T} \leq \frac{\psi^{-1 / \sigma}}{A \varphi^{\alpha}-\varphi(1+\delta)}
\end{aligned}
$$

for positive constants $\varphi, \psi, \phi, \zeta>0 .^{11}$

\footnotetext{
${ }^{11}$ Notice that these constants can be conveniently set in order to verify the conditions $\tilde{k}(x, 0)<$ $k(x, 0)<\hat{k}(x, 0)$ and $\tilde{\lambda}(x, T)<\Psi_{1}^{\prime}(k(x, T), x)<\hat{\lambda}(x, T)$.
} 
As stated in Theorem 1 we also have to ensure several boundary and continuity requirements (Assumptions 1-4). It is easy to see that Assumption 1 holds if we consider non-explosive initial distributions for the capital and the upper and lower solutions. Since $f_{1}$ and $f_{2}$ are continuously differentiable functions, they are Hölder continuous as well. Then, Assumption 4 is verified too. With respect to Assumption 2, this is guaranteed if we focus on interior non-explosive solutions. Notice lastly that $f_{1}$ is increasing in both $k$ and $\lambda .{ }^{12}$ One can also prove that $f_{2}$ is decreasing in $\lambda$. However, $f_{2}$ is increasing in $k$ since $\lambda>0$ and the production function is concave in $k$. We should then choose $\underline{a}_{1} \geq 0$ and $\bar{c}_{1} \leq 0$ according to Assumption 3. Moreover, since $f_{2}$ is increasing in $k$ but decreasing in $\lambda$, we can set $\bar{c}_{1}=0$ in order to ensure that Assumption 2 holds.

Summing-up, we have identified an upper and a lower solution. Since the assumptions of Theorem 1 hold for the functional forms of this illustration, we can then confirm that there exist a unique solution to our problem. Considering finally Theorem 2, we can guarantee that every solution lies between the upper and lower solutions, so we are not missing any additional solution. Hence, we can state the following proposition:

Proposition 1. The spatial growth model (12)-(13) is well-posed.

\subsubsection{Numerical application}

Let us consider the initial set $(\tilde{S})$ or $(\hat{S})$ in Theorem 1. Each of these sets defines the initialization of an algorithm starting from the lower or the upper solution, respectively. Our numerical method iteratively applies (11) in order to generate the sequences stated in the theorem. Notice that, since we have proved that both sequences converge, the algorithm can be initialized either with the lower or with the upper solution. The details of the algorithm are provided in Appendix D.

For this numerical example we consider the same functional forms as in the analytical application. Our method involves functions $F_{1}$ and $F_{2}$ defined in (9), so we have to specify $\underline{a}_{1}$ and $\bar{c}_{2}$. Taking into account the Assumptions 2 and 3 , one can verify that $\underline{a}_{1}=\delta$ and $\bar{c}_{2}=\delta+\rho$ satisfy all the requirements since we have set $\bar{c}_{1}=0$. All parameters values used in the simulations are displayed in Table 1. For the sake of illustration we set the scale parameter $A=1$. Regarding to the CRRA utility, Attanasio and Weber (1993) and Attanasio and Browning (1995) identify an interval for $\sigma$ between 1.25 and

\footnotetext{
${ }^{12} \partial f_{1} / \partial \lambda>0$ because $\lambda(x, t)=U^{\prime}(c(x, t))>0$. For $\partial f_{1} / \partial k>0$ the scale parameter $A$ should be high enough, i.e., $A>\delta\left(\varphi e^{\phi T}\right)^{1-\alpha} / \alpha$.
} 
3.33 using micro household data. Consistently with this range, Barro et al. (1995) assume $\sigma=2$. Chetty (2006) however observes that, even if macroeconomics tends to suggest values of $\sigma$ greater than in microeconomic studies, there is an upper bound implying that $\sigma<2$. Therefore, in line with these papers we choose for our application $\sigma=1.95$. As in Barro et al. (1995) and Barro and Sala-i-Martin (2004), we consider a time discount rate $\rho=0.02$ and a depreciation rate $\delta=0.05$. We set the output elasticity $\alpha=0.75$ following the notion of a broad measure that also includes knowledge capital (see, for instance, Barro and Sala-i-Martin, 2004; and Acemoglu, 2009). For the scrapping function, we choose a marginal value $\chi=0.1$ in order to reduce the direct welfare gains due to the capital left at the end of the decision horizon. Finally we set the constant $\epsilon$ of the lower solution to 1 . Indeed, as observed before, the iterations can start with either the upper or the lower solutions. Since for this model the lower solutions are simpler (see analytical application), we decide to initialize the algorithm with the set $(\tilde{S}) \cdot{ }^{13}$

\begin{tabular}{lll}
\hline$A$ & Scale parameter & 1 \\
$\sigma$ & Utility parameter & 1.95 \\
$\rho$ & Time discount rate & 0.02 \\
$\delta$ & Depreciation rate & 0.05 \\
$\alpha$ & Output elasticity & 0.75 \\
$\chi$ & Scrap parameter & 0.1 \\
$\epsilon$ & Lower solution constant & 1 \\
\hline
\end{tabular}

Table 1: Parameters values

For this illustration we assume a decision horizon $T=10$, considering that the region $\Omega$ of space is a real line of length 10 . Since our numerical method requires that both time and space are discretized, we divide them into 100 and 20 points respectively. So following the notation of Appendix D, $J=100$ and $N=20$ with $D t=0.1$ and $D x=0.5$. The economic aim of this application is to investigate the role played by capital mobility, paying particular attention to the evolution of spatial inequalities across locations. We will then assume that locations are spatially heterogenous with respect to their capital endowment. We specifically consider that the initial distribution of capital is given by

\footnotetext{
${ }^{13}$ As robustness checks we repeated simulations for $\alpha=1 / 3$, which is a value frequently assumed for a restricted definition of capital. We also tried alternative marginal scrap values $\chi=\{0.001,0.01,0.5,1\}$. In all these inspections we get similar qualitative results. We additionally verified that the simulated trajectories remain identical for different lower solutions, with $\epsilon=\{0.1,0.5,1.5,2\}$.
} 
$k_{0}(x)=164-40 \log (x)$ for $x$ in our discretized space $\Omega \simeq\{D x, 2 * D x, \ldots, N * D x\}$. Consequently, the endowment of capital stock is assumed to range from 164 to 44.17 as we move away from the origin.

We first consider the case of perfect capital mobility. For this scenario the algorithm needs 528 iterations to converge, with an Euclidean distance between two last iterations lower than 0.1 for both $k$ and $\lambda$. We plot in Figure 1 the optimal trajectories for the capital and its shadow price. We compare this case with the other extreme situation where there is no spatial diffusion. The policy maker still maximises the utility in the whole space. However, capital is not allowed to move from one location to another. For this simulation the algorithm needs 50 iterations to converge and, as in the previous case, the Euclidean distance between two iterations is lower than 0.1. Figure 2 reproduces the corresponding simulations.
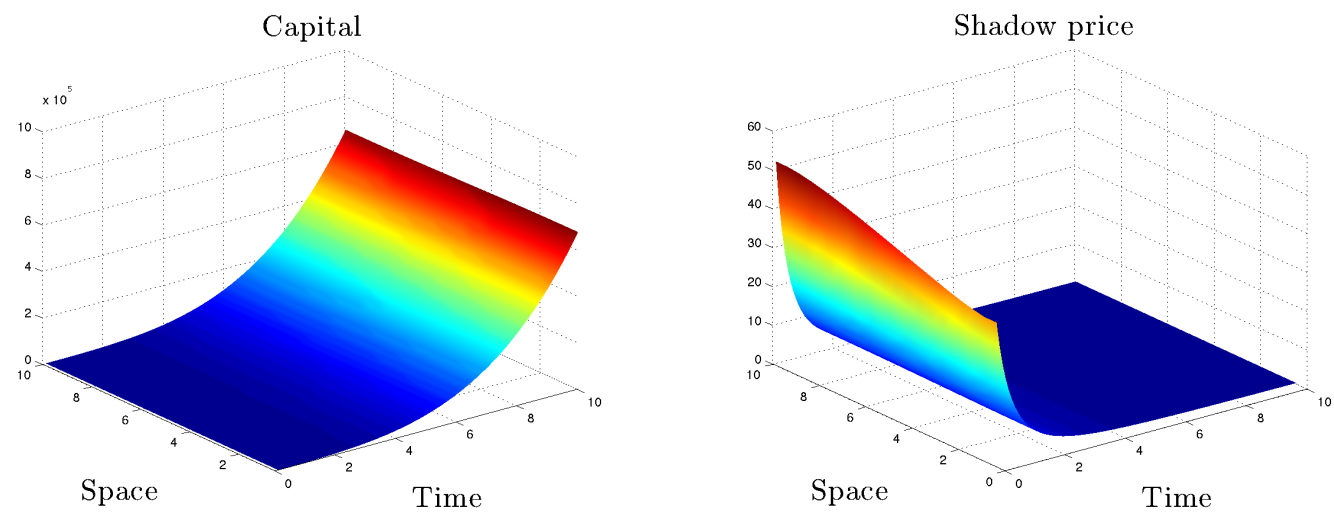

Figure 1: Spatial mobility

We can see from these figures that the economy does not immediately adjust even if capital is perfectly mobile. This outcome contrasts with the open-economy neoclassical growth model introduced in Barro et al. (1995). Transitional dynamics is a desirable property for a realistic economic model. However they observe that, under the reasonable assumptions of capital irreversibility and economic openness, perfect capital mobility is only compatible with this property if we include capital adjustment costs. Nevertheless, an important limitation of their set-up is that space is not explicitly included. We show in this regard that adding spacial structure, represented in our framework by the diffusion process, avoids immediate adjustment in the growth model even if capital is perfectly mobile. This conclusion is in line with Boucekkine et 

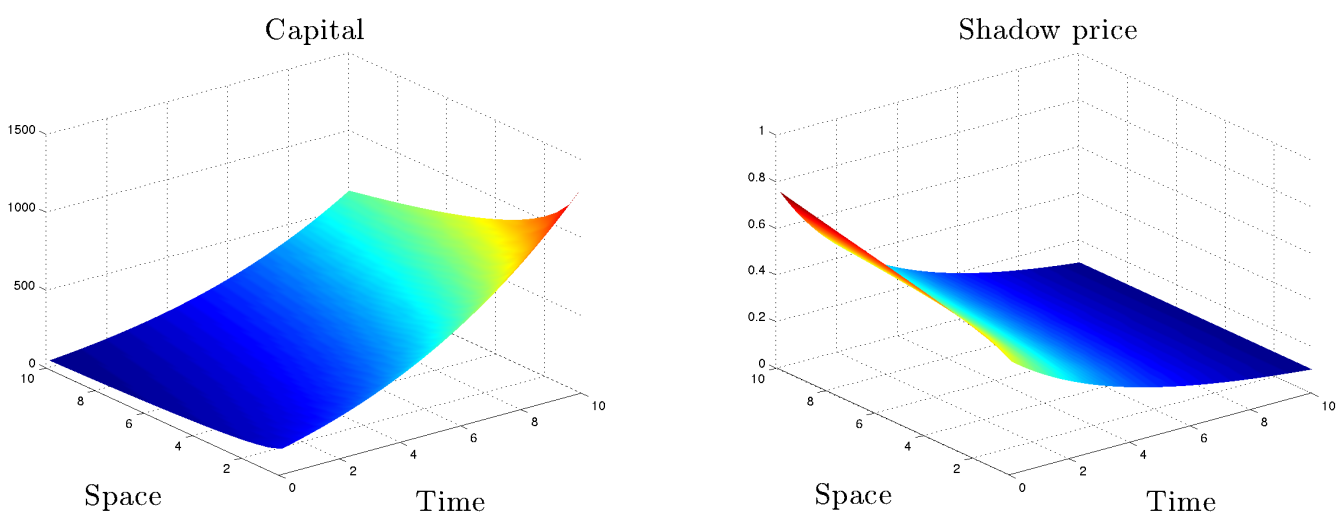

Figure 2: No spatial mobility

al. (2013a), where transitional dynamics arises in the AK growth model with explicit spatial structure. Within the context of Barro et al. (1995)'s observation, geography introduces an additional adjustment cost for capital.

Another remarkable feature that our model points out is the effect of capital mobility on social welfare. Capital mobility allows the economy to reduce the negative effect of diminishing returns to capital. When capital is mobile, it flows to locations with lower abundance (and, consequently, with higher shadow price). Then, the global impact of decreasing returns to scale reduces. As we can see in our simulations, capital increases with time in the two scenarios. But at the end of the decision horizon, both the minimum and the maximum stock of capital in the space are greater when there is spatial mobility (without mobility, $\left.\min \right|_{\Omega}(k(x, T))=611.93$ and $\left.\max \right|_{\Omega}(k(x, T))=1312.9$; while $\left.\min \right|_{\Omega}(k(x, T))=6.55 \times 10^{5}$ and $\left.\max \right|_{\Omega}(k(x, T))=6.74 \times 10^{5}$ with mobility $)$. Taking the evolution of the shadow price, we compute $c(x, t)$ since $U^{\prime}(c(x, t))=\lambda(x, t)$. We can therefore compare the social welfare in each type of economy, considering as well $k(x, T)$ and the corresponding scrap value. We find that capital mobility provides greater social welfare to the economy because capital can be spatially reallocated, thus inducing efficiency gains. This result is consistent with the importance that economic integration gives to capital mobility. Indeed, the elimination of any restrictions on the movement of capital is a primary objective of, for instance, the Economic and Monetary Union of the EU (EU, 2008; and, for a survey of the literature, see Kose et al., 2010).

Let us complete the illustration with the evolution of income inequality in the economy, considering the effect of capital mobility. The income of each location at time 
$t$ is defined as $y(x, t)=A f(k(x, t))$. Taking the simulated trajectories for $k(x, t)$, we can analyze the dynamics of the spatial income distribution by means of computing the Lorentz curve and the Gini coefficient of the economy in each time $t$. Figure 3 represents the evolution of spatial inequality, with and without capital mobility.

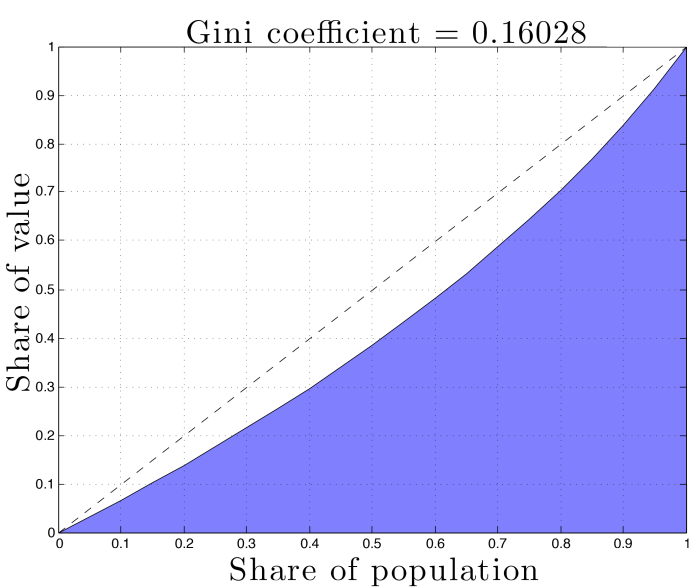

(a) Initial spatial inequality

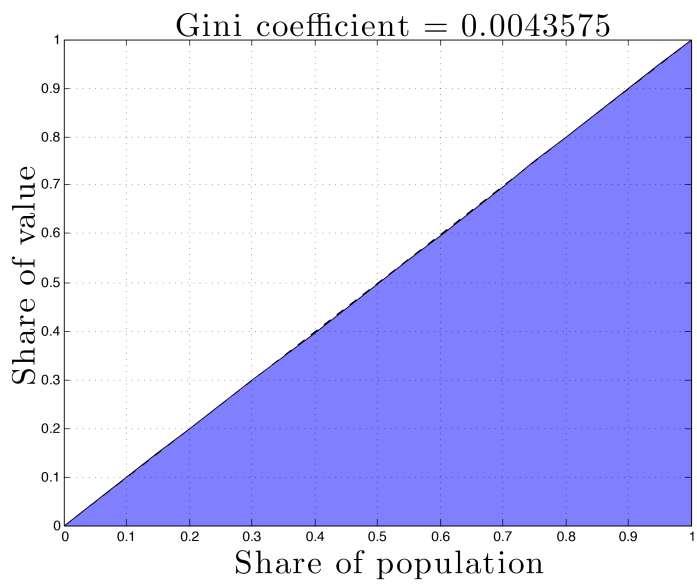

(c) Final spatial inequality: mobility

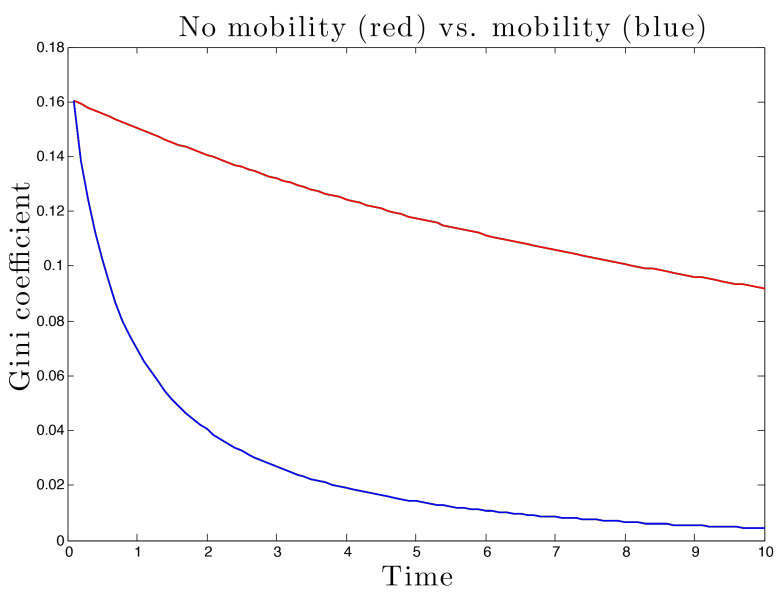

(b) Evolution of Gini index

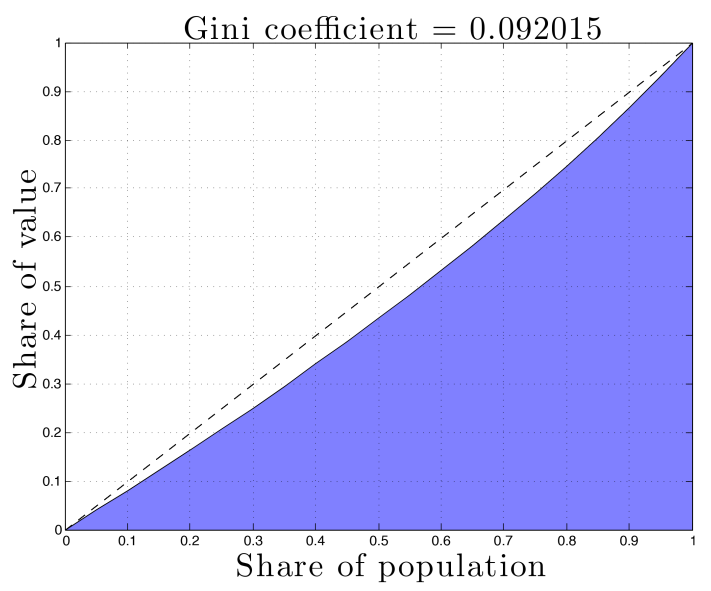

(d) Final spatial inequality: no mobility

Figure 3: Income distribution

Since we have assumed an initial distribution of capital that is spatially heterogeneous, Figure 3(a) depicts the resulting income inequality that corresponds to a Gini coefficient of 0.16 . In both scenarios, due to the decreasing returns to scale, inequality reduces as capital accumulates (see Figure 3b). However, the spatial mobility of capital allows the economy to achieve lower inequality (blue line). This is also clear if we evaluate the level of inequality in the economy at the end of the decision horizon. Figures $3(\mathrm{c})$ and $(\mathrm{d})$ show that the area between the line of equality (i.e., 45 degree line) and the Lorentz curve is smaller when capital is spatially mobile. The Gini coefficient is 0.004 
when the capital is mobile, which contrasts with a higher value (around 0.1) for the case where spatial mobility is not allowed. Consistently with the statement presented in papers such as Galor (1996) and Quah (1996a,b), our simulations show that improving capital mobility reduces the importance of the initial differences among locations. As before, the higher social value of capital in locations with lower abundance induces the spatial flow of this input.

\subsection{Land use dynamics and the environment}

Our last example is based on the spatial dynamic model introduced by Camacho and Pérez-Barahona (2015). As observed in the introduction, their set-up applies the spatialRamsey framework to study the social optimal use of land taking into account, in particular, the spread of local pollutants.

We assume an economy where each location has a unit of land, which can be devoted to three different activities: production, housing and pollution abatement. For simplicity, the space required for housing at each location is equal to its population density $f(x)$. We also consider no population growth. In this economy there exists a unique consumption good, which only requires land as a production input. We denote the production by $F(l)$. Production generates pollution. However, the remainder of the land can be used as pollution abatement with a technology $G(1-l-f(x))$. The policy maker chooses the level of consumption per capita and the use of land at each location that maximize the discounted social welfare of the entire population. An important feature of this problem is that, even if land is immobile by nature, local actions affect the whole space because pollution flows across locations. We describe the spatial dynamics of pollution $p(x, t)$ by means of the Gaussian plume model presented in Section 2 (equation 4$)$.

The policy maker maximizes the aggregate welfare in a bounded region of space $R$ over a finite time period $[0, T]$, where $c(x, t)$ denotes the consumption per capita at location $x$ and time $t$ :

$$
\max _{\{c, l\}} \int_{0}^{T} \int_{R} u(c(x, t)) f(x) e^{-\rho t} d x d t+\int_{R} \psi(p)(x, T) e^{-\rho T} d x
$$


subject to

$$
\left\{\begin{array}{l}
p_{t}(x, t)-p_{x x}(x, t)=P(p, x) A(x, t) F(l(x, t))-G(1-l-f(x)), \\
\int_{R} c(x, t) f(x) d x=\int_{R} P(p, x) A(x, t) F(l) d x, \\
\lim _{x \rightarrow \delta R} p_{x}(x, t)=0, \\
p(x, 0)=p_{0}(x) \geq 0 .
\end{array}\right.
$$

Following Camacho and Pérez-Barahona (2015), we introduce two discounts in the objective function (14). The term $e^{-\rho t}$ is the usual temporal discount with $\rho>0$. The spatial discount $f(x)$ represents the weight that the policy maker gives to each location. This can be identified, for instance, as the population density in order to avoid any subjective spatial preferences. In contrast to the application considered in the previous section, the scrap value represents this time the policy maker's concern about the state of pollution at the end of the planning horizon. Since pollution is assumed to reduce social welfare, the scrap function will be considered as negative.

In this economy pollution damages production. If we denote by $A(x, t)$ the total factor productivity at location $x$ at time $t$, we have that this location produces $P(p, x) A(x, t) F(l)$ units of final good when it devotes an amount $l$ of land to production. $1-P$ represents the share of foregone production due to pollution, with a function $P(p, x)$ decreasing in pollution. The first equation in (15) is the Gaussian plume, where the corresponding emissions $E(x, t)$ represents the instantaneous net emissions of each location, i.e., $P A F(l)-G$. As in Camacho and Pérez-Barahona (2015), the second constraint in (15) allows for the possibility of spatial reallocation of production. Hence, the policy maker collects all the production and reallocate it across locations at no cost. The limit constraint of the problem is the usual boundary condition: it is assumed that there is no pollution flow in the boundaries of the space. Finally $p(0, x)$ represents the initial spatial distribution of pollution. ${ }^{14}$

\footnotetext{
${ }^{14}$ Notice that our example focuses on local pollutants: the production in $(x, t)$ is negatively affected by $p(x, t)$. Camacho and Pérez-Barahona (2015) provides a more general framework, allowing for both local and global $\left(\int_{R} p(x, t) d x\right)$ effects of pollution. The later would add an integral equation to $(I)$.
} 
The Pontryagin conditions of the problem are:

$$
\left\{\begin{array}{l}
p_{t}(x, t)-p_{x x}(x, t)=P(p, x) A(x, t) F(l(x, t))-G(1-l-f(x)), \\
q_{t}(x, t)+q_{x x}(x, t)+P^{\prime}(p, x) A(x, t) F(l)\left[u^{\prime}(c(x, t))+q(x, t)\right]+\rho q=0, \\
{\left[u^{\prime}(c(x, t))+q(x, t)\right] P(p, x) A(x, t) F^{\prime}(l)+q(x, t) G^{\prime}(1-l-f(x))=0,} \\
\int_{R} c(x, t) f(x) d x=\int_{R} P(p, x) A(x, t) F(l) d x, \\
p(x, 0)=p_{0}(x) \geq 0, \\
\lim _{x \rightarrow \delta R} p_{x}(x, t)=0, \quad \lim _{x \rightarrow \delta R} p(x, t) q_{x}(x, t)=0, \\
q(x, T)=\psi^{\prime}(x, T),
\end{array}\right.
$$

for $(x, t) \in R \times[0, T]$.

As in Boucekkine et al. (2009), we should observe that the boundary condition for the shadow price $q(x, t)$ becomes the standard $\lim _{x \rightarrow \delta R} q_{x}(x, t)=0$ if we focus on interior solutions. Moreover, the last expression in (16) is the terminal condition of the problem. It states that, at the end of the planning horizon, the shadow price of pollution is equal to the marginal concern of the policy maker about the pollution left behind.

Camacho and Pérez-Barahona (2015) show that, due to the possibility of consumption "imports" (represented in this framework by the consumption reallocation), consumption is spatially homogenous. It is then possible to prove that $c(x, t)=c(t)=$ $\int_{R} P(p, x) A(x, t) F(l) d x / \int_{R} f(x) d x$. Moreover, replacing this expression in the equation describing the trade-off between consumption and pollution (third equation in 16), one can prove that the optimal land $l(x, t)$ is a unique function of $p(x, t)$ and $q(x, t)$. Consequently, the spatial dynamic behaviour of this economy is described by a set of equations that correspond to our general system $(I)$ in Section 3. We should notice though that our general formulation assumes that both state and co-state variables are positive. Therefore, we do the change of variable $\tilde{q}(x, t)=-q(x, t)$ since the shadow price of pollution is negative.

Let us apply Theorems 1 and 2 in order to study the existence of the unique solution to this economy. One can verify that the assumptions of both theorems hold for the functional forms considered in their paper. We should also observe that $f_{1}$ and $f_{2}$ are decreasing functions in both arguments $p$ and $\tilde{q}$. Then, we have to set $\underline{a}_{2} \leq 0$ and $\bar{c}_{2} \geq 0$. The last condition that we have to ensure is the existence of an upper and a lower solution to the problem. Camacho and Pérez-Barahona (2015) adapt Pao's stationary method (Theorem 3.4 in Pao, 1992) to study the existence of a unique time- 
invariant solution. In order to apply their result, they identify time-invariant upper and lower solutions. These stationary solutions are just particular cases of upper and lower solutions, which can be applied as well to the case of local pollutants. Hence, we can directly use our theorems, concluding that the problem has a unique solution:

Proposition 2. The land use dynamic problem (14)-(15) is well-possed.

\section{Concluding remarks}

We have proposed a general method to study the optimal solution of dynamic models based on the spatial Ramsey framework. The iterative nature of this approach also allowed us to provide a new algorithm to simulate the evolution in time and space of the economy. We included two economic illustrations of our technique. First, from an analytical point of view, we proved the existence of the unique optimal solution in spatial Ramsey-type models. We applied this result to the spatial growth model and to the optimal land-use set-up of Camacho and Pérez-Barahona (2015) for the spread of local pollutants. We concluded that both spatial-dynamic problems are well-posed. Considering the spatial growth model, the second illustration numerically investigated the role played by capital mobility in economic growth. We underline it effect on the dynamics of the economy. In particular, for plausible values of the parameters, capital mobility benefits social welfare reducing as well the spatial income inequality of the economy.

As in the existing mathematical literature on highly non-linear PDE, we focused on finite decision horizons. Considering a time span that is finite allows us for instance to be able to reverse time, which is a necessary step in this technique. In our approach the decision horizon can be as large as one wishes. However, it is worth paying attention to the problem of infinite horizon too. This case, even if beyond the scope of the paper, is particularly relevant to understanding the asymptotic behaviour of the economy. Boucekkine et al. (2013b) observes in this respect that infinite time horizon is still an open question even in the mathematical literature. Among other things, determining an appropriate limit transversality condition would be a key ingredient to address this question.

Several extensions can be made to our paper. The decentralization of the social optimum in spatial Ramsey-type models has not been explored yet in the literature. This would introduce the possibility of implementing public policy schemes that take 
into account the geographical characteristics of the economy. From this perspective, and in line with papers considering the interaction between capital mobility and tax coordination (among others, Bental, 1985; and Rodrik and van Ypersele, 2001; and Baldwin and Krugman, 2004), our approach would be useful to discuss the optimal degree of capital mobility in the economy. Another possible extension would incorporate human capital into the spatial growth model. As observed in the numerical application, we consider a broad definition of capital that includes knowledge as well. This is the case of tradable knowledge capital through, for instance, patents. But one could also think about the spread of human capital, which is reasonably less mobile (Galor, 1996; and Baldwin and Martin, 2004). Since our set-up admits multiple state variables, we could thus distinguish between physical and human capital. The spatio-temporal motion of each stock would be described by means of a PDE, assuming a lower diffusion degree for human capital.

\section{Appendices}

\section{A Pontryagin optimal conditions}

Let us define the value function associated to our problem:

$$
\begin{aligned}
& V\left(c, u_{1}, u_{2}\right) \equiv \int_{0}^{T} \int_{\Omega} U(c(x, t)) g(x, t) d x d t+\int_{\Omega} \Psi\left(u_{1}\right)(x, T) d x \\
& -\int_{0}^{T} \int_{\Omega} u_{2}(x, t)\left[u_{1, t}(x, t)-u_{1, x x}(x, t)-f_{1}\left(u_{1}, u_{2}\right)\right] d x d t
\end{aligned}
$$

where $u_{2}$ is an auxiliary function. $V$ is a function of $c, u_{1}$ and $u_{2}$. If there exists an optimal solution $\left(c^{*}, u_{1}^{*}, u_{2}^{*}\right)$, then any other solution to problem (1)-(2) can be written as a deviation from the optimal solution as

$$
\begin{aligned}
& c(x, t)=c^{*}(x, t)+\epsilon \kappa(x, t), \\
& u_{1}(x, t)=u_{1}^{*}(x, t)+\epsilon v_{1}(x, t), \\
& u_{2}(x, t)=u_{2}^{*}(x, t)+\epsilon v_{2}(x, t),
\end{aligned}
$$

for $\epsilon \in \mathbb{R}$. We can take the first order derivative of the value function $V$ with respect to $\epsilon$ in order to minimize the deviation of the trajectory from the optimal. Beforehand 
and using integration by parts, we rearrange some integral terms in $V$ :

$$
\begin{gathered}
\int_{0}^{T} \int_{\Omega} u_{2}(x, t) u_{1, x x}(x, t) d x d t=\left.\int_{0}^{T} u_{2}(x, t) u_{1, x}(x, t)\right|_{\delta \Omega} d t-\left.\int_{0}^{T} u_{2, x}(x, t) u_{1}(x, t)\right|_{\delta \Omega} d t \\
+\int_{0}^{T} \int_{\Omega} u_{2, x x}(x, t) u_{1}(x, t) d x d t
\end{gathered}
$$

and as usual:

$$
\begin{aligned}
& \int_{0}^{T} \int_{\Omega} u_{2}(x, t) u_{1, t}(x, t) d x d t=\left.\int_{\Omega} u_{1}(x, t) u_{2}(x, t)\right|_{0} ^{T} d x-\int_{0}^{T} \int_{\Omega} u_{1}(x, t) u_{2, t}(x, t) d x d t \\
& =\int_{\Omega} u_{1}(x, T) u_{2}(x, T) d x-\int_{\Omega} u_{1}(x, 0) u_{2}(x, 0) d x-\int_{0}^{T} \int_{\Omega} u_{2}(x, t) u_{2, t}(x, t) d x d t .
\end{aligned}
$$

We then obtain:

$$
\begin{aligned}
& \frac{\partial V\left(c, u_{1}, u_{2}\right)}{\partial \epsilon}=\int_{0}^{T} \int_{\Omega} U^{\prime}(c(x, t)) \kappa(x, t) g(x, t) d x d t+\int_{\Omega} \Psi_{1}^{\prime}\left(u_{1}(x, T)\right) v_{1}(x, T) d x \\
& +\int_{0}^{T} \int_{\Omega} v_{1}(x, t)\left[u_{2, t}(x, t)+u_{2, x x}(x, t)\right] d x d t \\
& -\int_{\Omega} u_{2}(x, T) v_{1}(x, T) d x-\left.\int_{0}^{T} v_{1}(x, t) u_{2, x}(x, t)\right|_{\delta \Omega} d t+\int_{0}^{T} \int_{\Omega} u_{2}(x, t)\left(\frac{\partial f_{1}}{\partial c} \kappa(x, t)+\frac{\partial f_{1}}{\partial u_{1}} v_{1}\right) d x d t .
\end{aligned}
$$

In order to get the necessary conditions, we can group the elements multiplying $\kappa$ and $v_{1}$, and equate them to zero. If all factors multiplying deviations from optimal values for $c$ and $u_{1}$ are equal to zero, then $\frac{\partial V}{\partial \epsilon}=0$. So we would need:

$$
\begin{cases}\kappa: & U^{\prime}(c) g(x, t)+u_{2} \frac{\partial f_{1}}{\partial c}=0, \\ v_{1}: & u_{2, t}+u_{2, x x}+\frac{\partial f_{1}}{\partial u_{1}} u_{2}=0 .\end{cases}
$$

The first equation in (21) shows that $c$ is an implicit function of $u_{1}$ and $u_{2}$. Moreover, we need to add to these conditions the following spatial boundary and transversality conditions:

$$
\left\{\begin{array}{l}
\lim _{x \rightarrow \delta \Omega} \quad u_{2, x}(x, t)=0, \\
u_{2}(x, T)=\Psi_{1}^{\prime}\left(u_{1}(x, T)\right) .
\end{array}\right.
$$

We then obtain the Pontryagin conditions $(O)$ by defining $f_{2}\left(u_{1}, u_{2}\right) \equiv-u_{2} \frac{\partial f_{1}}{\partial u_{1}}$. Finally, taking into account that $c$ can be expressed as an implicit function of $u_{1}$ and $u_{2}$, we can 
rewrite the set of necessary conditions as:

$$
\left\{\begin{array}{l}
u_{1, t}(x, t)-u_{1, x x}(x, t)=f_{1}\left(u_{1}, u_{2}\right) \\
u_{2, t}(x, t)+u_{2, x x}(x, t)=f_{2}\left(u_{1}, u_{2}\right) \\
\lim _{x \rightarrow \delta \Omega} u_{1, x}(x, t)=0 \\
\lim _{x \rightarrow \delta \Omega} u_{2, x}(x, t)=0 \\
u_{1}(x, 0)=u_{1,0}(x), \text { given}, \\
u_{2}(x, T)=\Psi_{1}^{\prime}\left(u_{1}(x, T)\right)
\end{array}\right.
$$

For the sake of exposition, we will use this alternative formulation of $(O)$ in Section 3.

\section{B Theorem 1 proof}

The final objective of this proof is to demonstrate the contraction property of $\mathbb{L}^{-1} F$. We can then conclude that the sequence

$$
\left(\begin{array}{c}
u_{1}^{k} \\
u_{2}^{k}
\end{array}\right)=\mathbb{L}^{-1} F\left(u^{k-1}\right)
$$

converges to the unique solution $\left(u_{1}, u_{2}\right)^{\prime}$. This outcome will require some intermediate results. Let us first summarize our method to build the sequences of the theorem. It mainly consists of four steps, which are developed in detail in the subsequent Proposition B1:

Step 1. We build a sequence of functions $\left\{\bar{u}_{1}^{k}\right\}_{k \in \mathbb{N}}$ starting from $\left(\bar{u}_{1}^{0}, \bar{u}_{2}^{0}\right)=\left(\hat{u}_{1}, \hat{u}_{2}\right)$. $\bar{u}_{1}^{1}$ is the solution to

$$
\left\{\begin{array}{l}
\mathbb{L}_{1} \bar{u}_{1}^{1}=F_{1}\left(\bar{u}_{1}^{0}, \bar{u}_{2}^{0}\right)=F_{1}\left(\hat{u}_{1}, \hat{u}_{2}\right), \forall(x, t) \in \Omega \times[0, T], \\
\lim _{x \rightarrow \delta \Omega} \bar{u}_{1, x}^{1}(x, t)=0, \forall t \geq 0,
\end{array}\right.
$$

with a suitable initial condition for $\bar{u}_{1}^{1}$ verifying that $u_{1}(x, 0) \leq \bar{u}_{1}^{1}(x, 0) \leq \hat{u}_{1}(x, 0)$. (22) together with the initial condition for $\bar{u}_{1}^{1}$ set up a linear parabolic PDE. Given the structure of the left hand side of the PDE in (22), and since $F_{1}\left(\hat{u}_{1}, \hat{u}_{2}\right)$ is a known bounded function of time and space, there exists a unique solution to (22) from $\bar{u}_{1}^{1}(x, 0)$ and subject to the boundary condition, as proven in Theorem 1.7 in Friedman (1959). Furthermore, this solution verifies that

$$
\int_{0}^{T} \int_{\Omega}\left|\bar{u}_{1}^{1}(x, t)\right| e^{-\varrho|x|^{2}} d x d t<\infty
$$


for some positive constant $\varrho$.

Step 2. We initiate the sequence $\left\{\bar{w}_{2}^{k}\right\}_{k \in \mathbb{N}}$ as $\bar{w}_{2}^{0}(x, t)=\hat{u}_{2}(x, T-t)$. Using $\bar{u}_{1}^{0}$ as $\bar{w}_{1}^{0}(x, t)=$ $\hat{u}_{1}^{1}(x, T-t), \bar{w}_{2}^{1}$ is the solution to

$$
\left\{\begin{array}{c}
\mathbb{L}_{2}^{\prime} \bar{w}_{2}^{1}=-F_{2}\left(\bar{w}_{1}^{0}, \bar{w}_{2}^{0}\right), \\
\lim _{x \rightarrow \delta \Omega} \bar{w}_{2}^{1}(x, t)=0,
\end{array}\right.
$$

and a suitable initial condition for $\bar{w}_{2}^{1}$ verifying $w_{2}(x, 0) \leq \bar{w}_{2}^{1}(x, 0) \leq \hat{w}_{2}(x, 0)$. As in Step 1, (23) is a linear parabolic PDE, which has a unique positive solution under the boundedness assumption on $\Psi$ and Assumption 1. Furthermore, the solution is bounded as before:

$$
\int_{0}^{T} \int_{\Omega}\left|\bar{w}_{2}^{1}(x, t)\right| e^{-\varrho|x|^{2}} d x d t<\infty
$$

for some positive constant $\varrho$. We proceed to the next iteration by undoing the change of variable $\bar{u}_{2}^{1}(x, t)=\bar{w}_{2}^{1}(x, T-t)$. Likewise, building on $\left(\bar{u}_{1}^{k-1}, \bar{u}_{2}^{k-1}\right)$, we compute $\left(\bar{u}_{1}^{k}, \bar{u}_{2}^{k}\right)$ following the procedure just described for $k=2,3, \cdots$. Moreover, for the subsequent iterations, we also need to state appropriate initial conditions $\bar{u}_{1}^{k}(x, 0)$ and $\bar{w}_{2}^{k}(x, 0)$ in order to ensure that the initial and terminal conditions in $(I)$ hold as $k \rightarrow \infty$ (notice that the terminal condition for $u_{2}$ is equivalent to the initial condition for $w_{2}$ after reversing time).

Step 3. In a similar manner, we can build a sequence $\left\{\underline{u}_{1}^{k}, \underline{u}_{2}^{k}\right\}_{k \in \mathbb{N}}$ and $\left\{\underline{w}_{1}^{k}, \underline{w}_{2}^{k}\right\}_{k \in \mathbb{N}}$ considering the lower solution.

Step 4. We prove that $\left\{\bar{u}_{1}^{k}, \bar{w}_{2}^{k}\right\}_{k \in \mathbb{N}}$ is monotonic non-increasing and that $\left\{\underline{u}_{1}^{k}, \underline{w}_{2}^{k}\right\}_{k \in \mathbb{N}}$ is monotonic non-decreasing. Undoing the change of time variable, we obtain the convergence of sequences $\left\{\bar{u}_{1}^{k}, \bar{u}_{2}^{k}\right\}_{k \in \mathbb{N}}$ and $\left\{\underline{u}_{1}^{k}, \underline{u}_{2}^{k}\right\}_{k \in \mathbb{N}}$ in $\Omega \times[0, T]$. As it will become clear in the proposition, the monotonicity and convergence of the generated sequence hinges on the choice of the initial conditions at each iteration. This choice will depend on the monotonicity characteristics of functions $F_{i}$.

We formally enunciate this iterative method as a proposition, together with the monotonicity property of the generated sequences:

Proposition B1. Let us consider the assumptions of the theorem. Let us introduce variables $w$ as the time-reverse of $u$, that is, $w_{i}(x, t) \equiv u_{i}(x, T-t), \hat{w}_{i}(x, t) \equiv \hat{u}_{i}(x, T-t)$ 
and $\tilde{w}_{i}(x, t) \equiv \tilde{u}_{i}(x, T-t)$ for $i=1,2$. We build sequences $\left\{\bar{u}_{1}^{k}, \bar{w}_{2}^{k}\right\}_{k \in \mathbb{N}}$ and $\left\{\underline{u}_{1}^{k}, \underline{w}_{2}^{k}\right\}_{k \in \mathbb{N}}$ following the process

$$
\left\{\begin{array}{l}
\mathbb{L}_{1} \bar{u}_{1}^{k}=F_{1}\left(\bar{u}_{1}^{k-1}, \bar{u}_{2}^{k-1}\right), \\
\bar{u}_{1}^{k}(x, 0)=\frac{\Upsilon}{k} \hat{u}_{1}(x, 0)+\left(1-\frac{\Upsilon}{k}\right) u_{1}(x, 0), \\
\lim _{x \rightarrow \delta \Omega} \bar{u}_{1, x}^{k}=0
\end{array}\right.
$$

and

$$
\left\{\begin{array}{l}
\mathbb{L}_{1} \underline{u}_{1}^{k}=F_{1}\left(\underline{u}_{1}^{k-1}, \underline{u}_{2}^{k-1}\right), \\
\underline{u}_{1}^{k}(x, 0)=\frac{\zeta}{k} \tilde{u}_{1}(x, 0)+\left(1-\frac{\zeta}{k}\right) u_{1}(x, 0), \\
\lim _{x \rightarrow \delta \Omega} \underline{u}_{1, x}^{k}=0,
\end{array}\right.
$$

with $0<\zeta<\Upsilon<1$,

$$
\left\{\begin{array}{l}
\mathbb{L}_{2}^{\prime} \bar{w}_{2}^{k}=-F_{2}\left(\bar{w}_{1}^{k-1}, \bar{w}_{2}^{k-1}\right), \\
\bar{w}_{2}^{k}(x, 0)=\frac{\psi}{k} \hat{w}_{2}(x, 0)+\left(1-\frac{\psi}{k}\right) \Psi_{1}^{\prime}\left(\bar{w}_{1}^{k-1}(x, 0), x\right), \\
\lim _{x \rightarrow \delta \Omega} \bar{w}_{2, x}^{k}=0
\end{array}\right.
$$

and

$$
\left\{\begin{array}{l}
\mathbb{L}_{2}^{\prime} \underline{w}_{2}^{k}=-F_{2}\left(\underline{w}_{1}^{k-1}, \underline{w}_{2}^{k-1}\right), \\
\underline{w}_{2}^{k}(x, 0)=\frac{\phi}{k} \tilde{w}_{2}(x, 0)+\left(1-\frac{\phi}{k}\right) \Psi_{1}^{\prime}\left(\underline{w}_{1}^{k-1}(x, 0), x\right), \\
\lim _{x \rightarrow \delta \Omega} \underline{w}_{2, x}^{k}=0,
\end{array}\right.
$$

with $0<\phi<\psi<1$. We initialize the sequences as

$$
\begin{aligned}
& \left(\bar{u}_{1}^{0}, \bar{u}_{2}^{0}\right)=\left(\hat{u}_{1}, \hat{u}_{2}\right), \\
& \left(\bar{w}_{1}^{0}, \bar{w}_{2}^{0}\right)=\left(\hat{w}_{1}, \hat{w}_{2}\right), \\
& \left(\underline{u}_{1}^{0}, \underline{u}_{2}^{0}\right)=\left(\tilde{u}_{1}, \tilde{u}_{2}\right), \\
& \left(\underline{w}_{1}^{0}, \underline{w}_{2}^{0}\right)=\left(\tilde{w}_{1}, \tilde{w}_{2}\right) .
\end{aligned}
$$

Then, the two sequences $\left\{\bar{u}_{1}^{k}, \bar{w}_{2}^{k}\right\}_{k \in \mathbb{N}}$ and $\left\{\underline{u}_{1}^{k}, \underline{w}_{2}^{k}\right\}_{k \in \mathbb{N}}$ obtained above have the monotone property

$$
\begin{aligned}
& \underline{u}_{1}^{k-1} \leq \underline{u}_{1}^{k} \leq \bar{u}_{1}^{k} \leq \bar{u}_{1}^{k-1} \\
& \underline{w}_{2}^{k-1} \leq \underline{w}_{2}^{k} \leq \bar{w}_{2}^{k} \leq \bar{w}_{2}^{k-1} .
\end{aligned}
$$


Proof. For the ease of presentation, we divide the proof in five parts. Along this proof it is important to keep in mind that

$$
\begin{aligned}
& \tilde{u}_{1}(x, t) \leq \hat{u}_{1}(x, t), \\
& \tilde{w}_{2}(x, t) \leq \hat{w}_{2}(x, t),
\end{aligned}
$$

and

$$
\begin{aligned}
& \tilde{u}_{1}(x, 0)<u_{1}(x, 0)<\hat{u}_{1}(x, 0), \\
& \tilde{w}_{2}(x, 0)<\Psi_{1}^{\prime}\left(\tilde{u}_{1}(x, T), x\right), \\
& \Psi_{1}^{\prime}\left(\hat{u}_{1}(x, T), x\right)<\hat{w}_{2}(x, 0) .
\end{aligned}
$$

i) Let us first construct $\left\{\bar{u}_{1}^{k}\right\}_{k \in \mathbb{N}}$ and $\left\{\bar{w}_{2}^{k}\right\}_{k \in \mathbb{N}}$. We initialize the sequences as

$$
\begin{aligned}
& \left(\bar{u}_{1}^{0}, \bar{u}_{2}^{0}\right)=\left(\hat{u}_{1}, \hat{u}_{2}\right), \\
& \left(\bar{w}_{1}^{0}, \bar{w}_{2}^{0}\right)=\left(\hat{w}_{1}, \hat{w}_{2}\right) .
\end{aligned}
$$

As we have observed in the short description of our iterative method, the first element of the sequence $\left\{\bar{u}_{1}^{k}\right\}_{k \in \mathbb{N}}$ is the solution to

$$
\left\{\begin{array}{l}
\mathbb{L}_{1} \bar{u}_{1}^{1}=F_{1}\left(\bar{u}_{1}^{0}, \bar{u}_{2}^{0}\right)=F_{1}\left(\hat{u}_{1}, \hat{u}_{2}\right), \\
\bar{u}_{1}^{1}(x, 0)=\Upsilon \hat{u}_{1}(x, 0)+(1-\Upsilon) u_{1}(x, 0), \\
\lim _{x \rightarrow \delta \Omega} \bar{u}_{1, x}^{1}=0 .
\end{array}\right.
$$

The PDE in $(24)$ has a unique solution $\bar{u}_{1}^{1}$ given that $F_{1}\left(\hat{u}_{1}, \hat{u}_{2}\right)$ is known, $\mathbb{L}_{1}$ is linear, and $\bar{u}_{1}^{1}(x, 0)$ is strictly positive. Next, we show that $\bar{u}_{1}^{0} \geq \bar{u}_{1}^{1}$. Let us construct an auxiliary variable $v_{1}^{0}$ on $\Omega \times[0, T]:^{15}$

$$
v_{1}^{0} \equiv \bar{u}_{1}^{0}-\bar{u}_{1}^{1}=\hat{u}_{1}-\bar{u}_{1}^{1} .
$$

Then,

$$
\begin{gathered}
\mathbb{L}_{1} v_{1}^{0}=\mathbb{L}_{1}\left(\bar{u}_{1}^{0}-\bar{u}_{1}^{1}\right)=\hat{u}_{1, t}-\hat{u}_{1, x x}+\underline{a}_{1}(x, t) \hat{u}_{1}-F_{1}\left(\hat{u}_{1}, \hat{u}_{2}\right) \\
=\hat{u}_{1, t}-\hat{u}_{1, x x}+\underline{a}_{1}(x, t) \hat{u}_{1}-f_{1}\left(\hat{u}_{1}, \hat{u}_{2}\right)-\underline{a}_{1}(x, t) \hat{u}_{1} \geq 0 .
\end{gathered}
$$

We know that the expression above is non-negative because $\left(\hat{u}_{1}, \hat{u}_{2}\right)$ is an upper solution of problem $(I)$. Besides,

$$
v_{1}^{0}(x, 0)=\bar{u}_{1}^{0}(x, 0)-\bar{u}_{1}^{1}(x, 0)=\hat{u}_{1}(x, 0)-\Upsilon \hat{u}_{1}(x, 0)-(1-\Upsilon) u_{1}(x, 0)
$$

\footnotetext{
${ }^{15} \mathrm{We}$ deliberately abuse of notation in this proof. For $i=1,2$, we denote by $v_{i}^{0}, v_{i}^{1}$ and $v_{i}^{k}$ different auxiliary variables. Since the usage of these variables is unambiguously restricted to the proof of each intermediate result, we choose this option for the sake of presentation.
} 


$$
=(1-\Upsilon)\left(\hat{u}_{1}(x, 0)-u_{1}(x, 0)\right)>0 .
$$

Then, invoking classical results on positiveness as in Pao (1992), we conclude that $v_{1}^{0}(x, t) \geq 0$, which implies that $\bar{u}_{1}^{0}(x, t) \geq \bar{u}_{1}^{1}(x, t)$ for all $(x, t) \in \Omega \times[0, T]$.

Let us similarly consider $\bar{w}_{2}^{1}$, solution to

$$
\left\{\begin{array}{l}
\mathbb{L}_{2}^{\prime} \bar{w}_{2}^{1}=-F_{2}\left(\bar{w}_{1}^{0}, \bar{w}_{2}^{0}\right)=-F_{2}\left(\hat{w}_{1}, \hat{w}_{2}\right), \\
\bar{w}_{2}^{1}(x, 0)=\psi \hat{w}_{2}(x, 0)+(1-\psi) \Psi_{1}^{\prime}\left(\bar{w}_{1}^{0}(x, 0), x\right), \\
\lim _{x \rightarrow \delta \Omega} \bar{w}_{2, x}^{1}=0,
\end{array}\right.
$$

and construct $v_{2}^{0} \equiv \bar{w}_{2}^{0}-\bar{w}_{2}^{1}=\hat{w}_{2}-\bar{w}_{2}^{1}$, where $\hat{w}_{2}(x, t)=\hat{u}_{2}(x, T-t)$ is the time reverse of the second coordinate of the upper solution $\left(\hat{u}_{1}, \hat{u}_{2}\right)$. We prove a similar result for $v_{2}^{0}$ :

$$
\begin{gathered}
\mathbb{L}_{2}^{\prime} v_{2}^{0}=\mathbb{L}_{2}^{\prime}\left(\hat{w}_{2}-\bar{w}_{2}^{1}\right)=\hat{w}_{2, t}-\hat{w}_{2, x x}+\bar{c}_{2}(x, t) \hat{w}_{2}-F_{2}\left(\hat{w}_{1}, \hat{w}_{2}\right) \\
=\hat{w}_{2, t}-\hat{w}_{2, x x}+\bar{c}_{2}(x, t) \hat{w}_{2}+f_{2}\left(\hat{w}_{1}, \hat{w}_{2}\right)-\bar{c}_{2}(x, t) \hat{w}_{2} \geq 0,
\end{gathered}
$$

because of (8). We need to check the sign of $v_{2}^{0}(x, 0)$. Using the definition of the initial conditions:

$$
\begin{gathered}
v_{2}^{0}(x, 0)=\hat{w}_{2}(x, 0)-\bar{w}_{2}^{1}(x, 0)=\hat{w}_{2}(x, 0)-\psi \hat{w}_{2}(x, 0)-(1-\psi) \Psi_{1}^{\prime}\left(\bar{w}_{1}^{0}(x, 0), x\right) \\
=(1-\psi)\left(\hat{w}_{2}(x, 0)-\Psi_{1}^{\prime}\left(\hat{w}_{1}(x, 0), x\right)\right)>0 .
\end{gathered}
$$

Then $v_{2}^{0}(x, t) \geq 0 \Rightarrow \hat{w}_{2}(x, t) \geq \bar{w}_{2}^{1}(x, t)$ for every $(x, t) \in \Omega \times[0, T]$.

ii) We apply the same procedure to the other sequences, starting instead with the lower solutions:

$$
\begin{aligned}
& \left(\underline{u}_{1}^{0}, \underline{u}_{2}^{0}\right)=\left(\tilde{u}_{1}, \tilde{u}_{2}\right), \\
& \left(\underline{w}_{1}^{0}, \underline{w}_{2}^{0}\right)=\left(\tilde{w}_{1}, \tilde{w}_{2}\right) .
\end{aligned}
$$

$\underline{u}_{1}^{1}$ is the solution to

$$
\left\{\begin{array}{l}
\mathbb{L}_{1} \underline{u}_{1}^{1}=F_{1}\left(\underline{u}_{1}^{0}, \underline{u}_{2}^{0}\right)=F_{1}\left(\tilde{u}_{1}, \tilde{u}_{2}\right), \\
\underline{u}_{1}^{1}(x, 0)=\zeta \tilde{u}_{1}(x, 0)+(1-\zeta) u_{1}(x, 0), \\
\lim _{x \rightarrow \delta \Omega} \underline{u}_{1, x}^{1}=0 .
\end{array}\right.
$$

Then, defining $v_{1}^{0}$ and $v_{2}^{0}$ as

$$
\begin{aligned}
& v_{1}^{0} \equiv \underline{u}_{1}^{1}-\underline{u}_{1}^{0}=\underline{u}_{1}^{1}-\tilde{u}_{1}, \\
& v_{2}^{0} \equiv \underline{w}_{2}^{1}-\underline{w}_{2}^{0}=\underline{w}_{2}^{1}-\tilde{w}_{2},
\end{aligned}
$$


we can prove that $\underline{u}_{1}^{1} \geq \underline{u}_{1}^{0}$ and $\underline{w}_{2}^{1} \geq \underline{w}_{2}^{0}$. Let us consider in this respect $v_{1}^{0}=$ $\underline{u}_{1}^{1}-\underline{u}_{1}^{0}=\underline{u}_{1}^{1}-\tilde{u}_{1}$. Applying the operator $\mathbb{L}_{1}$ :

$$
\begin{aligned}
\mathbb{L}_{1} v_{1}^{0} & =\mathbb{L}_{1}\left(\underline{u}_{1}^{1}-\tilde{u}_{1}\right)=F_{1}\left(\underline{u}_{1}^{0}, \underline{u}_{2}^{0}\right)-\left(\tilde{u}_{1, t}-\tilde{u}_{1, x x}+\bar{c}_{1} \tilde{u}_{1}\right) \\
& =f_{1}\left(\tilde{u}_{1}, \tilde{u}_{2}\right)+\bar{c}_{1} \tilde{u}_{1}-\tilde{u}_{1, t}+\tilde{u}_{1, x x}-\bar{c}_{1} \tilde{u}_{1} \geq 0,
\end{aligned}
$$

because $\left(\tilde{u}_{1}, \tilde{u}_{2}\right)$ is a lower solution. Regarding to the initial condition:

$$
\begin{gathered}
v_{1}^{0}(x, 0)=\underline{u}_{1}^{1}(x, 0)-\underline{u}_{1}^{0}(x, 0)=\underline{u}_{1}^{1}(x, 0)-\tilde{u}_{1}(x, 0) \\
=\zeta \tilde{u}_{1}(x, 0)+(1-\zeta) u_{1}(x, 0)-\tilde{u}_{1}(x, 0)=(1-\zeta)\left(u_{1}(x, 0)-\tilde{u}_{1}(x, 0)\right)>0 .
\end{gathered}
$$

Then, we conclude that $\underline{u}_{1}^{1} \geq \underline{u}_{1}^{0}$.

Similarly, let us prove that $\underline{w}_{2}^{0} \leq \underline{w}_{2}^{1}$. Taking the definition of $v_{2}^{0}$ we can show that

$$
\begin{aligned}
\mathbb{L}_{2}^{\prime} v_{2}^{0} & =\mathbb{L}_{2}^{\prime}\left(\underline{w}_{2}^{1}-\underline{w}_{2}^{0}\right)=-F_{2}\left(\underline{w}_{1}^{0}, \underline{w}_{2}^{0}\right)-\left(\underline{w}_{2, t}-\underline{w}_{2, x x}+\bar{c}_{2} \underline{w}_{2}\right) \\
& =-f_{2}\left(\tilde{w}_{1}, \tilde{w}_{2}\right)+\bar{c}_{2} \tilde{w}_{2}-\left(\tilde{w}_{2, t}-\tilde{w}_{2, x x}+\bar{c}_{2} \tilde{w}_{2}\right) \geq 0 .
\end{aligned}
$$

Moreover,

$$
\begin{gathered}
v_{2}^{0}(x, 0)=\underline{w}_{2}^{1}(x, 0)-\underline{w}_{2}^{0}(x, 0) \\
=\underline{w}_{2}^{1}(x, 0)-\tilde{w}_{2}(x, 0)=\phi \tilde{w}_{2}(x, 0)+(1-\phi) \Psi_{1}^{\prime}\left(\underline{w}_{1}^{0}(x, 0), x\right)-\tilde{w}_{2}(x, 0) \\
=(1-\phi)\left(\Psi_{1}^{\prime}\left(\tilde{w}_{1}(x, 0), x\right)-\tilde{w}_{2}(x, 0)\right)>0 .
\end{gathered}
$$

We therefore confirm our statement.

iii) We prove next that $\underline{u}_{1}^{1} \leq \bar{u}_{1}^{1}$ and $\underline{w}_{2}^{1} \leq \bar{w}_{2}^{1}$. To do so, we define the auxiliary variables $v_{1}^{1}, v_{2}^{1}$ on $\Omega \times[0, T]$ as

$$
\begin{aligned}
& v_{1}^{1} \equiv \bar{u}_{1}^{1}-\underline{u}_{1}^{1}, \\
& v_{2}^{1} \equiv \bar{w}_{2}^{1}-\underline{w}_{2}^{1} .
\end{aligned}
$$

Let us show first that $\bar{u}_{1}^{1} \leq \underline{u}_{1}^{1}$. Applying the operator $\mathbb{L}_{1}$,

$$
\begin{gathered}
\mathbb{L}_{1} v_{1}^{1}=\mathbb{L}_{1}\left(\bar{u}_{1}^{1}-\underline{u}_{1}^{1}\right)=F_{1}\left(\hat{u}_{1}, \hat{u}_{2}\right)-F_{1}\left(\tilde{u}_{1}, \tilde{u}_{2}\right) \\
=f_{1}\left(\hat{u}_{1}, \hat{u}_{2}\right)-f_{1}\left(\tilde{u}_{1}, \tilde{u}_{2}\right)+\underline{a}_{1}\left(\hat{u}_{1}-\tilde{u}_{1}\right) .
\end{gathered}
$$

Since $\hat{u}_{1} \geq \tilde{u}_{1}$, we can see that $\mathbb{L}_{1} v_{1}^{1} \geq 0$ if $\underline{a}_{1} \geq 0$ and $f_{1}$ increasing in both arguments $u_{1}$ and $u_{2}$; or if alternatively $\underline{a}_{2} \leq 0$. In the later case, we would have

$$
\mathbb{L}_{1} v_{1}^{1}=f_{1}\left(\hat{u}_{1}, \hat{u}_{2}\right)-f_{1}\left(\tilde{u}_{1}, \tilde{u}_{2}\right)+\underline{a}_{1}\left(\hat{u}_{1}-\tilde{u}_{1}\right) \geq-\underline{a}_{2}\left(\hat{u}_{2}-\tilde{u}_{2}\right) \geq 0 .
$$


Taking into account that

$$
\begin{gathered}
v_{1}^{1}(x, 0)=\bar{u}_{1}^{1}(x, 0)-\underline{u}_{1}^{1}(x, 0)=\Upsilon \hat{u}_{1}(x, 0)+(1-\Upsilon) u_{1}(x, 0)-\left(\zeta \tilde{u}_{1}(x, 0)+(1-\zeta) u_{1}(x, 0)\right) \\
=\Upsilon \hat{u}_{1}(x, 0)-\zeta \tilde{u}_{1}(x, 0)-(\Upsilon-\zeta) u_{1}(x, 0) \\
\geq \Upsilon \hat{u}_{1}(x, 0)-\zeta \hat{u}_{1}(x, 0)-(\Upsilon-\zeta) u_{1}(x, 0)=(\Upsilon-\zeta)\left(\hat{u}_{1}(x, 0)-u_{1}(x, 0)\right)>0,
\end{gathered}
$$

we conclude that $v_{1}^{1}(x, t) \geq 0, \forall(x, t) \in \Omega \times[0, T]$. Hence, $\underline{u}_{1}^{1} \leq \bar{u}_{1}^{1}$.

$i v$ ) Let us consider $v_{2}^{1}$. Applying $\mathbb{L}_{2}^{\prime}$,

$$
\begin{gathered}
\mathbb{L}_{2}^{\prime} v_{2}^{1}=\mathbb{L}_{2}^{\prime}\left(\bar{w}_{2}^{1}-\underline{w}_{2}^{1}\right)=-F_{2}\left(\hat{w}_{1}, \hat{w}_{2}\right)+F_{2}\left(\tilde{w}_{1}, \tilde{w}_{2}\right) \\
=-f_{2}\left(\hat{w}_{1}, \hat{w}_{2}\right)+f_{2}\left(\tilde{w}_{1}, \tilde{w}_{2}\right)+\bar{c}_{2}\left(\hat{w}_{2}-\tilde{w}_{2}\right),
\end{gathered}
$$

which can be positive if either $f_{2}$ decreasing in both $w_{1}$ and $w_{2}$ and $\bar{c}_{2} \geq 0$; or if $\overline{c_{1}} \leq 0$ whatever the sign of the partial derivatives of $f_{2}$ since

$$
\mathbb{L}_{2}^{\prime} v_{2}^{1} \geq-\overline{c_{1}}\left(\hat{w}_{1}-\tilde{w}_{1}\right) \geq 0
$$

Moreover, one can verify that

$$
\begin{gathered}
v_{2}^{1}(x, 0)=\bar{w}_{2}^{1}(x, 0)-\underline{w}_{2}^{1}(x, 0) \\
=\psi \hat{w}_{2}(x, 0)+(1-\psi) \Psi_{1}^{\prime}\left(\bar{w}_{1}^{0}(x, 0), x\right)-\phi \tilde{w}_{2}(x, 0)-(1-\phi) \Psi_{1}^{\prime}\left(\underline{w}_{1}^{0}(x, 0), x\right) \\
\geq(\psi-\phi)\left(\hat{w}_{2}(x, 0)-\Psi_{1}^{\prime}\left(\hat{w}_{1}(x, 0), x\right)\right)>0 .
\end{gathered}
$$

We therefore conclude that $v_{2}^{1} \geq 0$ and $\bar{w}_{2}^{1} \geq \underline{w}_{2}^{1}$.

$v$ ) We have proven so far that

$$
\underline{u}_{1}^{0} \leq \underline{u}_{1}^{1} \leq \bar{u}_{1}^{1} \leq \bar{u}_{1}^{0}
$$

and

$$
\underline{w}_{2}^{0} \leq \underline{w}_{2}^{1} \leq \bar{w}_{2}^{1} \leq \bar{w}_{2}^{0}
$$

These two conclusions allow us to identify the required base case to show by mathematical induction that our sequences are ordered. Following this strategy, let us assume that

$$
\underline{u}_{1}^{k-1} \leq \underline{u}_{1}^{k} \leq \bar{u}_{1}^{k} \leq \bar{u}_{1}^{k-1}
$$

and

$$
\underline{w}_{2}^{k-1} \leq \underline{w}_{2}^{k} \leq \bar{w}_{2}^{k} \leq \bar{w}_{2}^{k-1}
$$


We have to show, as inductive step, that

$$
\underline{u}_{1}^{k} \leq \underline{u}_{1}^{k+1} \leq \bar{u}_{1}^{k+1} \leq \bar{u}_{1}^{k}
$$

and

$$
\underline{w}_{2}^{k} \leq \underline{w}_{2}^{k+1} \leq \bar{w}_{2}^{k+1} \leq \bar{w}_{2}^{k} .
$$

Let us begin by verifying that the inequality $\bar{u}_{1}^{k+1} \leq \bar{u}_{1}^{k}$ holds. We define in this regard the auxiliary variable $v_{1}^{k}$ as

$$
v_{1}^{k} \equiv \bar{u}_{1}^{k}-\bar{u}_{1}^{k+1}
$$

Applying the operator $\mathbb{L}_{1}$,

$$
\begin{gathered}
\mathbb{L}_{1} v_{1}^{k}=F_{1}\left(\bar{u}_{1}^{k-1}, \bar{u}_{2}^{k-1}\right)-F_{1}\left(\bar{u}_{1}^{k}, \bar{u}_{2}^{k}\right) \\
=f_{1}\left(\bar{u}_{1}^{k-1}, \bar{u}_{2}^{k-1}\right)-f_{1}\left(\bar{u}_{1}^{k}, \bar{u}_{2}^{k}\right)+\underline{a}_{1}\left(\bar{u}^{k-1}-\bar{u}_{1}^{k}\right) \geq 0 .
\end{gathered}
$$

Since $\bar{u}_{1}^{k} \leq \bar{u}_{1}^{k-1}$ and $\bar{u}_{2}^{k} \leq \bar{u}_{2}^{k-1}$, we can see that $\mathbb{L}_{1} v_{1}^{k} \geq 0$ if $\underline{a}_{1} \geq 0$ and $f_{1}$ increasing in both arguments; or if alternatively $\underline{a}_{1} \leq 0$. In the later case,

$$
\mathbb{L}_{1} v_{1}^{k}=f_{1}\left(\bar{u}_{1}^{k-1}, \bar{u}_{2}^{k-1}\right)-f_{1}\left(\bar{u}_{1}^{k}, \bar{u}_{2}^{k}\right)+\underline{a}_{1}\left(\bar{u}^{k-1}-\bar{u}_{1}^{k}\right) \geq-\underline{a}_{2}\left(\bar{u}_{2}^{k-1}-\bar{u}_{2}^{k}\right) \geq 0 .
$$

Moreover,

$$
\begin{gathered}
v_{1}^{k}(x, 0)=\bar{u}_{1}^{k}(x, 0)-\bar{u}_{1}^{k+1}(x, 0) \\
=\frac{\Upsilon}{k} \hat{u}_{1}(x, 0)+\left(1-\frac{\Upsilon}{k}\right) u_{1}(x, 0)-\frac{\Upsilon}{k+1} \hat{u}_{1}(x, 0)-\left(1-\frac{\Upsilon}{k+1}\right) u_{1}(x, 0) \\
=\Upsilon \hat{u}_{1}(x, 0)\left(\frac{1}{k}-\frac{1}{k+1}\right)-\Upsilon u_{1}(x, 0)\left(\frac{1}{k}-\frac{1}{k+1}\right), \\
=\Upsilon\left(\frac{1}{k}-\frac{1}{k+1}\right)\left(\hat{u}_{1}(x, 0)-u_{1}(x, 0)\right)>0,
\end{gathered}
$$

since $\hat{u}_{1}(x, 0)>u_{1}(x, 0)$. Hence $v_{1}^{k} \geq 0$ and $\bar{u}_{1}^{k} \geq \bar{u}_{1}^{k+1}$.

Similarly, let us prove the inequality $\underline{u}_{1}^{k} \leq \underline{u}_{1}^{k+1}$. We define the auxiliary variable $v_{1}^{k}$ as

$$
v_{1}^{k} \equiv \underline{u}_{1}^{k+1}-\underline{u}_{1}^{k} .
$$

Applying again $\mathbb{L}_{1}$,

$$
\begin{gathered}
\mathbb{L}_{1} v_{1}^{k}=F_{1}\left(\underline{u}_{1}^{k}, \underline{u}_{2}^{k}\right)-F_{1}\left(\underline{u}_{1}^{k-1}, \underline{u}_{2}^{k-1}\right) \\
=f_{1}\left(\underline{u}_{1}^{k}, \underline{u}_{2}^{k}\right)-f_{1}\left(\underline{u}_{1}^{k-1}, \underline{u}_{2}^{k-1}\right)+\underline{a}_{1}\left(\underline{u}_{1}^{k}-\underline{u}_{1}^{k-1}\right) \geq 0 .
\end{gathered}
$$


Since $\underline{u}_{1}^{k} \geq \underline{u}_{1}^{k-1}$ and $\underline{u}_{2}^{k} \geq \underline{u}_{2}^{k-1}$, we can see that $\mathbb{L}_{1} v_{1}^{1} \geq 0$ if $\underline{a}_{1} \geq 0$ and $f_{1}$ increasing in both arguments; or if alternatively $\underline{a}_{2} \leq 0$. In the later case, we would have

$$
\mathbb{L}_{1} v_{1}^{k}=f_{1}\left(\underline{u}_{1}^{k}, \underline{u}_{2}^{k}\right)-f_{1}\left(\underline{u}_{1}^{k-1}, \underline{u}_{2}^{k-1}\right)+\underline{a}_{1}\left(\underline{u}_{1}^{k}-\underline{u}_{1}^{k-1}\right) \geq-\underline{a}_{2}\left(\underline{u}_{2}^{k}-\underline{u}_{2}^{k-1}\right) \geq 0 .
$$

One can also verify that

$$
\begin{gathered}
v_{1}^{k}(x, 0)=\underline{u}_{1}^{k+1}(x, 0)-\underline{u}_{1}^{k}(x, 0) \\
=\frac{\zeta}{k+1} \tilde{u}_{1}(x, 0)+\left(1-\frac{\zeta}{k+1}\right) u_{1}(x, 0)-\frac{\zeta}{k} \tilde{u}_{1}(x, 0)+\left(1-\frac{\zeta}{k}\right) u_{1}(x, 0) \\
=\zeta \frac{1}{k(k+1)} \tilde{u}_{1}(x, 0)+\zeta \frac{1}{k(k+1)} u_{1}(x, 0) \\
=\zeta \frac{1}{k(k+1)}\left(u_{1}(x, 0)-\tilde{u}_{1}(x, 0)\right)>0 .
\end{gathered}
$$

Therefore $v_{1}^{k} \geq 0$ and $\underline{u}_{1}^{k} \leq \underline{u}_{1}^{k+1}$. Next, we need to prove that $\underline{u}_{1}^{k+1} \leq \bar{u}_{1}^{k+1}$. Let us define $v_{1}^{k+1} \equiv \bar{u}_{1}^{k+1}-\underline{u}_{1}^{k+1}$, then

$$
\mathbb{L}_{1} v_{1}^{k+1}=F_{1}\left(\bar{u}_{1}^{k}, \bar{u}_{2}^{k}\right)-F_{1}\left(\underline{u}_{1}^{k}, \underline{u}_{2}^{k}\right)=f_{1}\left(\bar{u}_{1}^{k}, \bar{u}_{2}^{k}\right)-f_{1}\left(\underline{u}_{1}^{k}, \underline{u}_{2}^{k}\right)+\underline{a}_{1}\left(\bar{u}_{1}^{k}-\underline{u}_{1}^{k}\right) \geq 0 .
$$

Since $\bar{u}_{1}^{k} \geq \underline{u}_{1}^{k}$ and $\bar{u}_{2}^{k} \geq \underline{u}_{2}^{k}$, we can see that $\mathbb{L}_{1} v_{1}^{k+1} \geq 0$ if $\underline{a}_{1} \geq 0$ and $f_{1}$ increasing in both arguments; or if alternatively $\underline{a}_{2} \leq 0$. In the later case,

$$
\mathbb{L}_{1} v_{1}^{k+1}=f_{1}\left(\bar{u}_{1}^{k}, \bar{u}_{2}^{k}\right)-f_{1}\left(\underline{u}_{1}^{k}, \underline{u}_{2}^{k}\right)+\underline{a}_{1}\left(\bar{u}_{1}^{k}-\underline{u}_{1}^{k}\right) \geq-\underline{a}_{2}\left(\bar{u}_{2}^{k}-\underline{u}_{2}^{k}\right) \geq 0 .
$$

We can verify that the initial distribution of $v_{1}^{k+1}$ is positive:

$$
\begin{gathered}
v_{1}^{k+1}(x, 0)=\bar{u}_{1}^{k+1}(x, 0)-\underline{u}_{1}^{k+1}(x, 0) \\
=\frac{\Upsilon}{k+1} \hat{u}_{1}(x, 0)+\left(1-\frac{\Upsilon}{k+1}\right) u_{1}(x, 0)-\frac{\zeta}{k+1} \tilde{u}_{1}(x, 0)-\left(1-\frac{\zeta}{k+1}\right) u_{1}(x, 0) \\
=\frac{\Upsilon}{k+1} \hat{u}_{1}(x, 0)-\frac{\zeta}{k+1} \tilde{u}_{1}(x, 0)-\frac{\Upsilon-\zeta}{k+1} u_{1}(x, 0) \geq \frac{\Upsilon-\zeta}{k+1} \hat{u}_{1}(x, 0)-\frac{\Upsilon-\zeta}{k+1} u_{1}(x, 0) \\
=\frac{\Upsilon-\zeta}{k+1}\left(\hat{u}_{1}(x, 0)-u_{1}(x, 0)\right)>0,
\end{gathered}
$$

Hence $v_{1}^{k+1} \geq 0$ and $\underline{u}_{1}^{k+1} \leq \bar{u}_{1}^{k+1}$. Summarizing, we can conclude that

$$
\underline{u}_{1}^{k} \leq \underline{u}_{1}^{k+1} \leq \bar{u}_{1}^{k+1} \leq \bar{u}_{1}^{k} .
$$

Let us consider the second set of inequalities. Defining $v_{2}^{k} \equiv \bar{w}_{2}^{k}-\bar{w}_{2}^{k+1}$, we apply the operator $\mathbb{L}_{2}^{\prime}$ :

$\mathbb{L}_{2}^{\prime} v_{2}^{k}=F_{2}\left(\bar{w}_{1}^{k}, \bar{w}_{2}^{k}\right)-F_{2}\left(\bar{w}_{1}^{k-1}, \bar{w}_{2}^{k-1}\right)=f_{2}\left(\bar{w}_{1}^{k}, \bar{w}_{2}^{k}\right)-f_{2}\left(\bar{w}_{1}^{k-1}, \bar{w}_{2}^{k-1}\right)+\bar{c}_{2}\left(\bar{w}_{2}^{k-1}-\bar{w}_{2}^{k}\right) \geq 0$. 
Since $\bar{w}_{1}^{k} \leq \bar{w}_{1}^{k-1}$ and $\bar{w}_{2}^{k} \leq \underline{w}_{2}^{k-1}$, we can see that $\mathbb{L}_{2}^{\prime} v_{2}^{k} \geq 0$ if $\bar{c}_{2} \geq 0$ and $f_{2}$ decreasing in both arguments; or if alternatively $\bar{c}_{1} \leq 0$. In the later case,

$$
\begin{gathered}
\mathbb{L}_{2}^{\prime} v_{2}^{k}=f_{2}\left(\bar{w}_{1}^{k}, \bar{w}_{2}^{k}\right)-f_{2}\left(\bar{w}_{1}^{k-1}, \bar{w}_{2}^{k-1}\right)+\bar{c}_{2}\left(\bar{w}_{2}^{k-1}-\bar{w}_{2}^{k}\right) \\
\geq-\bar{c}_{1}\left(\bar{w}_{1}^{k-1}-\bar{w}_{1}^{k}\right) \geq 0 .
\end{gathered}
$$

Moreover,

$$
\begin{gathered}
v_{2}^{k}(x, 0)=\bar{w}_{2}^{k}(x, 0)-\bar{w}_{2}^{k+1}(x, 0) \\
=\psi\left(\frac{1}{k}-\frac{1}{k+1}\right) \hat{w}_{2}(x, 0)+\left(1-\frac{\psi}{k}\right) \Psi_{1}^{\prime}\left(\bar{w}_{1}^{k-1}(x, 0), x\right)-\left(1-\frac{\psi}{k+1}\right) \Psi_{1}^{\prime}\left(\bar{w}_{1}^{k}(x, 0), x\right) .
\end{gathered}
$$

Since $\bar{w}_{1}^{k+1}(x, 0) \leq \bar{w}_{1}^{k}(x, 0)$, for a convex function $\Psi$,

$$
\begin{gathered}
w_{2}^{k}(x, 0) \\
\geq \psi\left(\frac{1}{k}-\frac{1}{k+1}\right) \hat{w}_{2}(x, 0)+\left(1-\frac{\psi}{k}\right) \Psi_{1}^{\prime}\left(\bar{w}_{1}^{k-1}(x, 0), x\right)-\left(1-\frac{\psi}{k+1}\right) \Psi_{1}^{\prime}\left(\bar{w}_{1}^{k-1}(x, 0), x\right) \\
=\psi\left(\frac{1}{k}-\frac{1}{k+1}\right)\left[\hat{w}_{2}(x, 0)-\Psi_{1}^{\prime}\left(\bar{w}_{1}^{k-1}(x, 0), x\right)\right] \\
>\psi\left(\frac{1}{k}-\frac{1}{k+1}\right)\left[\hat{w}_{2}(x, 0)-\Psi_{1}^{\prime}\left(\hat{w}_{1}(x, 0), x\right)\right]>0 .
\end{gathered}
$$

Hence, we conclude that $v_{2}^{k}(x, 0)>0$ and $\bar{w}_{2}^{k+1} \leq \bar{w}_{2}^{k}$.

We prove next that $\underline{w}_{2}^{k} \leq \underline{w}_{2}^{k+1}$. Let us define $v_{2}^{k} \equiv \underline{w}_{2}^{k+1}-\underline{w}_{2}^{k}$. Considering the operator $\mathbb{L}_{2}^{\prime}$ we obtain

$$
\begin{gathered}
\mathbb{L}_{2}^{\prime} v_{2}^{k}=F_{2}\left(\underline{w}_{1}^{k-1}, \underline{w}_{2}^{k-1}\right)-F_{2}\left(\underline{w}_{1}^{k}, \underline{w}_{2}^{k}\right) \\
=f_{2}\left(\underline{w}_{1}^{k-1}, \underline{w}_{2}^{k-1}\right)-f_{2}\left(\underline{w}_{1}^{k}, \underline{w}_{2}^{k}\right)+\bar{c}_{2}\left(\underline{w}_{2}^{k}-\underline{w}_{2}^{k-1}\right) \geq 0 .
\end{gathered}
$$

Since $\underline{w}_{1}^{k-1} \leq \underline{w}_{1}^{k}$ and $\underline{w}_{2}^{k-1} \leq \underline{w}_{2}^{k}$, we can see that $\mathbb{L}_{2}^{\prime} v_{2}^{k} \geq 0$ if $\bar{c}_{2} \geq 0$ and $f_{2}$ decreasing in both arguments; or if alternatively $\bar{c}_{1} \leq 0$. In the later case,

$$
\begin{gathered}
\mathbb{L}_{2}^{\prime} v_{2}^{k}=f_{2}\left(\underline{w}_{1}^{k-1}, \underline{w}_{2}^{k-1}\right)-f_{2}\left(\underline{w}_{1}^{k}, \underline{w}_{2}^{k}\right)+\bar{c}_{2}\left(\underline{w}_{2}^{k}-\underline{w}_{2}^{k-1}\right) \\
\geq-\bar{c}_{1}\left(\underline{w}_{1}^{k}-\underline{w}_{1}^{k-1}\right) \geq 0 .
\end{gathered}
$$

We check the initial distribution of $v_{2}^{k}$, for a convex function $\Psi$ :

$$
\begin{gathered}
v_{2}^{k}(x, 0)=\underline{w}_{2}^{k+1}(x, 0)-\underline{w}_{2}^{k}(x, 0) \\
=\frac{\phi}{k+1} \tilde{w}_{2}(x, 0)+\left(1-\frac{\phi}{k+1}\right) \Psi_{1}^{\prime}\left(\underline{w}_{1}^{k}(x, 0), x\right)-\frac{\phi}{k} \tilde{w}_{2}(x, 0)-\left(1-\frac{\phi}{k}\right) \Psi_{1}^{\prime}\left(\underline{w}_{1}^{k-1}(x, 0), x\right)
\end{gathered}
$$


$\geq \phi \frac{1}{k(k+1)}\left(\Psi_{1}^{\prime}\left(\underline{w}_{1}^{k}(x, 0), x\right)-\tilde{w}_{2}(x, 0)\right)>\phi \frac{1}{k(k+1)}\left(\Psi_{1}^{\prime}\left(\tilde{w}_{1}(x, 0), x\right)-\tilde{w}_{2}(x, 0)\right)>0$,

Therefore $v_{2}^{k}(x, 0)>0$ and $\underline{w}_{2}^{k} \leq \underline{w}_{2}^{k+1}$.

Let us finally show that $\underline{w}_{2}^{k+1} \leq \bar{w}_{2}^{k+1}$. This time we define $w_{2}^{k+1} \equiv \bar{w}_{2}^{k+1}-\underline{w}_{2}^{k+1}$. Applying $\mathbb{L}_{2}^{\prime}$,

$$
\begin{gathered}
\mathbb{L}_{2}^{\prime} v_{2}^{k+1}=-F_{2}\left(\bar{w}_{1}^{k}, \bar{w}_{2}^{k}\right)+F_{2}\left(\underline{w}_{1}^{k}, \underline{w}_{2}^{k}\right) \\
=-f_{2}\left(\bar{w}_{1}^{k}, \bar{w}_{2}^{k}\right)+f_{2}\left(\underline{w}_{1}^{k}, \underline{w}_{2}^{k}\right)+\bar{c}_{2}\left(\bar{w}_{2}^{k}-\underline{w}_{2}^{k}\right) \geq-\bar{c}_{1}\left(\bar{w}_{1}^{k}-\underline{w}_{1}^{k}\right) \geq 0 .
\end{gathered}
$$

Since $\bar{w}_{2}^{k} \geq \underline{w}_{2}^{k}$ and $\bar{w}_{1}^{k} \geq \underline{w}_{1}^{k}$, we can see that $\mathbb{L}_{2}^{\prime} v_{2}^{k+1} \geq 0$ if $\bar{c}_{2} \geq 0$ and $f_{2}$ decreasing in both arguments; or if alternatively $\bar{c}_{1} \leq 0$. In the later case,

$$
\mathbb{L}_{2}^{\prime} v_{2}^{k+1}=-f_{2}\left(\bar{w}_{1}^{k}, \bar{w}_{2}^{k}\right)+f_{2}\left(\underline{w}_{1}^{k}, \underline{w}_{2}^{k}\right)+\bar{c}_{2}\left(\bar{w}_{2}^{k}-\underline{w}_{2}^{k}\right) \geq-\bar{c}_{1}\left(\bar{w}_{1}^{k}-\underline{w}_{1}^{k}\right) \geq 0 .
$$

Regarding the initial condition $v_{2}^{k+1}(x, 0)$ :

$$
\begin{gathered}
w_{2}^{k+1}(x, 0)= \\
=\frac{\psi}{k+1} \hat{w}_{2}(x, 0)+\left(1-\frac{\psi}{k+1}\right) \Psi_{1}^{\prime}\left(\bar{w}_{1}^{k}(x, 0), x\right)-\frac{\phi}{k+1} \tilde{w}_{2}(x, 0)-\left(1-\frac{\phi}{k+1}\right) \Psi_{1}^{\prime}\left(\underline{w}_{1}^{k}(x, 0), x\right) \\
\geq \frac{\psi-\phi}{k+1} \hat{w}_{2}(x, 0)-\frac{\psi-\phi}{k+1} \Psi_{1}^{\prime}\left(\bar{w}_{1}^{k}(x, 0), x\right)=\frac{\psi-\phi}{k}\left(\hat{w}_{2}(x, 0)-\Psi_{1}^{\prime}\left(\bar{w}_{1}^{k}(x, 0), x\right)\right) \\
\geq \frac{\psi-\phi}{k}\left(\hat{w}_{2}(x, 0)-\Psi_{1}^{\prime}\left(\hat{w}_{1}(x, 0), x\right)\right)>0,
\end{gathered}
$$

for a convex function $\Psi$. This implies that $v_{2}^{k+1} \geq 0$ and hence $\underline{w}_{2}^{k+1} \leq \bar{w}_{2}^{k+1}$. So we can state that

$$
\underline{w}_{2}^{k} \leq \underline{w}_{2}^{k+1} \leq \bar{w}_{2}^{k+1} \leq \bar{w}_{2}^{k}
$$

Before going to the next intermediate result, it is important to consider the following observations regarding the generality of Proposition B1:

Remark 1. The initial conditions for $\bar{w}_{2}^{k}$ and $\underline{w}_{2}^{k}$ correspond to a function $\Psi$ that is assumed to be convex in $w_{1}$. For $\Psi_{1}$ concave we should initialize $\bar{w}_{2}^{k}$ and $\underline{w}_{2}^{k}$ as

$$
\begin{aligned}
& \bar{w}_{2}^{k}(x, 0)=\frac{\psi}{k} \hat{w}_{2}(x, 0)+\left(1-\frac{\psi}{k}\right) \Psi_{1}^{\prime}\left(\underline{w}_{1}^{k-1}(x, 0), x\right), \\
& \underline{w}_{2}^{k}(x, 0)=\frac{\phi}{k} \tilde{w}_{2}(x, 0)+\left(1-\frac{\phi}{k}\right) \Psi_{1}^{\prime}\left(\bar{w}_{1}^{k-1}(x, 0), x\right) .
\end{aligned}
$$


Remark 2. In the absence of an upper solution, it is still true that $\left\{\underline{u}_{1}^{k}, \underline{u}_{2}^{k}\right\}$ is monotone non-decreasing. Then the sequence either converges to some limit or it becomes unbounded, as $k \rightarrow \infty$. Nevertheless, we cannot prove that the limit is a solution of the original problem. The same holds true for the monotone non-increasing sequence $\left\{\bar{u}_{1}^{k}, \bar{u}_{2}^{k}\right\}$.

Remark 3. The initial conditions for our sequences have been set in order to ensure that the iterative process is monotone. Alternative forms might be considered. The main requirement is that they should converge towards the initial and terminal conditions of the original problem when $k \rightarrow \infty$.

This far we have constructed four sequences of continous functions $\left\{\bar{u}_{1}^{k}\right\}_{k \in \mathbb{N}},\left\{\underline{u}_{1}^{k}\right\}_{k \in \mathbb{N}}$, $\left\{\bar{w}_{2}^{k}\right\}_{k \in \mathbb{N}}$ and $\left\{\underline{w}_{2}^{k}\right\}_{k \in \mathbb{N}}$, which are monotone and bounded. One can then conclude that they converge uniformly. Hence, there exist functions $\underline{u}_{1}, \bar{u}_{1}, \underline{w}_{2}$ and $\bar{w}_{2}$ defined over $\Omega \times[0, T]$, such that, $\forall(x, t) \in \Omega \times[0, T]$,

$$
\begin{aligned}
& \lim _{\substack{k \rightarrow \infty \\
k \in \mathbb{N}}} \underline{u}_{1}^{k}(x, t)=\underline{u}_{1}(x, t), \\
& \lim _{\substack{k \rightarrow \infty \\
k \in \mathbb{N}}} \bar{u}_{1}^{k}(x, t)=\bar{u}_{1}(x, t),
\end{aligned}
$$

and

$$
\begin{aligned}
& \lim _{\substack{k \rightarrow \infty \\
k \in \mathbb{N}}} \underline{w}_{2}^{k}(x, t)=\underline{w}_{2}(x, t), \\
& \lim _{\substack{k \rightarrow \infty \\
k \in \mathbb{N}}} \bar{w}_{2}^{k}(x, t)=\bar{w}_{2}(x, t) .
\end{aligned}
$$

Notice that $\underline{u}_{1}(x, 0)=\bar{u}_{1}(x, 0)=u_{1}(x, 0)$ and $\underline{w}_{2}(x, 0)=\bar{w}_{2}(x, 0)=\Psi_{1}^{\prime}\left(u_{1}(x, T), x\right)$ (see Remark 3 above). Undoing the change of variable, we also obtain limit functions $\bar{u}_{2}(x, t)=\bar{w}_{2}(x, T-t)$ and $\underline{u}_{2}(x, t)=\underline{w}_{2}(x, T-t)$. The next question is whether the limit functions $\underline{u}_{1}(x, t), \bar{u}_{1}(x, t), \underline{u}_{2}(x, t)$ and $\bar{u}_{2}(x, t)$ coincide, i.e., whether for all $(x, t) \in \Omega \times[0, T]$ it is true that $\underline{u}_{1}(x, t)=\bar{u}_{1}(x, t)$ and $\underline{u}_{2}(x, t)=\bar{u}_{2}(x, t)$. At this point, we directly focus on $u_{1}$ and $u_{2}$. In order to prove that $\underline{u}_{1}=\bar{u}_{1}$ and $\underline{u}_{2}=\bar{u}_{2}$, we will show that the linear operators $\mathbb{L}_{1}$ and $\mathbb{L}_{2}$, and the corresponding diagonal matrix $\mathbb{L} \equiv \operatorname{diag}\left(\mathbb{L}_{1}, \mathbb{L}_{2}\right)$, are invertible. Then, after proving that $\mathbb{L}^{-1} F(u)$ is a contraction where $F(u) \equiv\left(F_{1}(u), F_{2}(u)\right)^{\prime}$, we can conclude the uniqueness of the limits. Let us first consider the existence of $\mathbb{L}^{-1}$ :

Lemma B1. There exists a real positive number $\gamma$ such that the inverse operators of $\mathbb{L}_{1}$ and $\mathbb{L}_{2}$, denoted by $\mathbb{L}_{1}^{-1}$ and $\mathbb{L}_{2}^{-1}$ respectively, exist and satisfy

$$
\left|\mathbb{L}_{i}^{-1} u_{i}-\mathbb{L}_{i}^{-1} u_{i}^{\prime}\right|_{0} \leq \gamma^{-1}\left|u_{i}-u_{i}^{\prime}\right|_{0},
$$


for any $(x, t) \in \Omega \times[0, T]$, where $|\cdot|_{0}$ is the supremum norm for functions in $R\left(\mathbb{L}_{i}\right)$, the range of $\mathbb{L}_{i}$ :

$$
\mathbb{L}_{i}: D\left(\mathbb{L}_{i}\right) \longrightarrow C(\Omega \times[0, T]),
$$

for $i=1,2$, where $\mathbb{L}_{i}$ 's domain is

$$
\begin{aligned}
& D\left(\mathbb{L}_{1}\right)=\left\{u_{1} \in C(\bar{\Omega} \times[0, T]) \cap C^{1,2}(\Omega \times[0, T]), \quad \lim _{x \rightarrow \delta \Omega} u_{1, x}=0, \quad u_{1}(x, 0)=u_{1,0}(x), \text { given }\right\}, \\
& D\left(\mathbb{L}_{2}\right)=\left\{u_{2} \in C(\bar{\Omega} \times[0, T]) \cap C^{1,2}(\Omega \times[0, T]), \lim _{x \rightarrow \delta \Omega} u_{2, x}=0, \quad u_{2}(x, T)=\Psi_{1}^{\prime}\left(u_{1}(x, T), x\right)\right\} .
\end{aligned}
$$

Proof. We will treat $\mathbb{L}_{1}$ and $\mathbb{L}_{2}$ separately. We begin by proving lemma's result for $\mathbb{L}_{1}$.

I. Let us take any two $u_{1}, u_{1}^{\prime} \in D\left(\mathbb{L}_{1}\right)$ verifying $u_{1} \neq u_{1}^{\prime}$, i.e., they do not coincide in all $(x, t) \in \Omega \times[0, T]$. We build $v \equiv u_{1}-u_{1}^{\prime}$, and let $\left(x_{1}, t_{1}\right) \in \Omega \times[0, T]$ be a point such that

$$
|v|_{0}=\left|v\left(x_{1}, t_{1}\right)\right|
$$

That is to say, $v$ attains the supremum of its absolute value at $\left(x_{1}, t_{1}\right)$. Let us show that

$$
v\left(x_{1}, t_{1}\right)\left(\mathbb{L}_{1} u_{1}\left(x_{1}, t_{1}\right)-\mathbb{L}_{1} u_{1}^{\prime}\left(x_{1}, t_{1}\right)\right) \geq \gamma_{1}|v|_{0}^{2},
$$

which would imply, following Pao (1992), the statement of the lemma for the operator $\mathbb{L}_{1}$. The inequality above is obvious if $v\left(x_{1}, t_{1}\right)=0$. If $v\left(x_{1}, t_{1}\right) \neq 0$ then $v\left(x_{1}, t_{1}\right)$ is a positive maximum or a negative minimum in $\bar{\Omega} \times[0, T]$.

We know that, for $t=0$ and for any $x \in \Omega, v(x, 0)=u_{1,0}(x)-u_{1,0}(x)=0$. Therefore $t_{1}>0$. Given that $t_{1}$ is an extremum,

$$
\left\{\begin{array}{l}
v_{t}\left(x_{1}, t_{1}\right)=0, \text { if } t_{1}<T, \\
\text { or } v\left(x_{1}, t_{1}\right) v_{t}\left(x_{1}, t_{1}\right) \geq 0, \text { if } t_{1}=T .
\end{array}\right.
$$

Regarding the space we also have two alternatives. Rather $x_{1}$ is in the interior of the space or it is located in the frontier, i.e., $x_{1} \in \stackrel{\Omega}{\Omega}$ or $x_{1} \in \delta \Omega$, respectively:

I.1. If $x_{1} \in \stackrel{\Omega}{\Omega}$, we have that

$$
v\left(x_{1}, t_{1}\right) v_{x x}\left(x_{1}, t_{1}\right) \leq 0
$$

because $\left(x_{1}, t_{1}\right)$ is a extremum. Considering together (25) and (26),

$$
v\left(x_{1}, t_{1}\right)\left[v_{t}\left(x_{1}, t_{1}\right)-v_{x x}\left(x_{1}, t_{1}\right)\right] \geq 0
$$

or, equivalently, rewriting the inequality above in terms of $u_{1}$ and taking into account the definition of $\mathbb{L}_{1}$,

$$
v\left(x_{1}, t_{1}\right)\left\{\mathbb{L}_{1} u_{1}\left(x_{1}, t_{1}\right)-\mathbb{L}_{1} u_{1}^{\prime}\left(x_{1}, t_{1}\right)-\underline{a}_{1}\left(x_{1}, t_{1}\right)\left[u_{1}\left(x_{1}, t_{1}\right)-u_{1}^{\prime}\left(x_{1}, t_{1}\right)\right]\right\} \geq 0 .
$$


Therefore, since $\underline{a}_{1}(x, t)$ is a bounded function (assumption of the theorem),

$$
\begin{gathered}
v\left(x_{1}, t_{1}\right)\left[\mathbb{L}_{1} u_{1}\left(x_{1}, t_{1}\right)-\mathbb{L}_{1} u_{1}^{\prime}\left(x_{1}, t_{1}\right)\right] \geq \underline{a}_{1}\left(x_{1}, t_{1}\right)\left|u_{1}\left(x_{1}, t_{1}\right)-u_{1}^{\prime}\left(x_{1}, t_{1}\right)\right|^{2} \\
\geq \gamma_{1}\left|v\left(x_{1}, t_{1}\right)\right|^{2}=\gamma_{1}|v|_{0}^{2}
\end{gathered}
$$

for any $\gamma_{1} \in \mathbb{R}$ such that $0<\gamma_{1} \leq \underline{a}_{1}\left(x_{1}, t_{1}\right)$. Hence, as in Pao (1992), since

$$
|v|_{0}\left|\mathbb{L}_{1} u_{1}-\mathbb{L}_{1} u_{1}^{\prime}\right|_{0} \geq v\left(x_{1}, t_{1}\right)\left[\mathbb{L}_{1} u_{1}\left(x_{1}, t_{1}\right)-\mathbb{L}_{1} u_{1}^{\prime}\left(x_{1}, t_{1}\right)\right],
$$

we can conclude that, using (27) and (28),

$$
\left|\mathbb{L}_{1} u_{1}-\mathbb{L}_{1} u_{1}^{\prime}\right|_{0} \geq \gamma_{1}|v|_{0}
$$

I.2. Let us consider the case where $x_{1}$ is in the frontier. If $x_{1} \in \delta \Omega$ then, for any $\epsilon>0$,

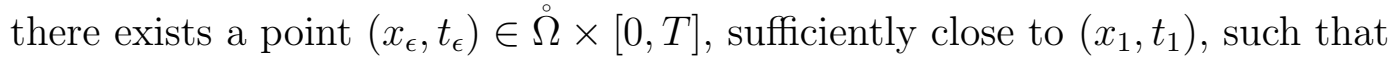

$$
\left\{\begin{array}{l}
v\left(x_{1}, t_{1}\right) v_{t}\left(x_{\epsilon}, t_{\epsilon}\right) \geq \frac{-\epsilon}{2}|v|_{0}^{2}, \\
v\left(x_{1}, t_{1}\right) v_{x x}\left(x_{\epsilon}, t_{\epsilon}\right) \leq \frac{-\epsilon}{2}|v|_{0}^{2},
\end{array}\right.
$$

where $t_{\epsilon} \equiv t_{1} \pm \epsilon$ and $x_{\epsilon} \equiv x_{1} \pm \epsilon$. We can state the first inequality because $\left(x_{1}, t_{1}\right)$ is a maximum of the absolute value. Hence, when we approach to $\left(x_{1}, t_{1}\right)$, we must observe that $v_{t} \rightarrow 0$ by continuity of the trajectories. The same is true for the derivative in space. As a consequence,

$$
v\left(x_{1}, t_{1}\right)\left[v_{t}\left(x_{\epsilon}, t_{\epsilon}\right)-v_{x x}\left(x_{\epsilon}, t_{\epsilon}\right)\right] \geq-\epsilon|v|_{0}^{2}
$$

and, as before,

$$
\left.v\left(x_{1}, t_{1}\right)\left(\mathbb{L}_{1} u_{1}-\mathbb{L}_{1} u_{1}^{\prime}\right)\right|_{\left(x_{\epsilon}, t_{\epsilon}\right)} \geq\left(\gamma_{1}-\epsilon\right)|v|_{0}^{2} .
$$

The arbitrariness of $\epsilon$ therefore ensures that

$$
\left|\mathbb{L}_{1} u_{1}\left(x_{1}, t_{1}\right)-\mathbb{L}_{1} u_{1}^{\prime}\left(x_{1}, t_{1}\right)\right| \geq \gamma_{1}\left|u_{1}\left(x_{1}, t_{1}\right)-u_{1}^{\prime}\left(x_{1}, t_{1}\right)\right|=\gamma_{1}\left|u_{1}-u_{1}^{\prime}\right|_{0}
$$

for $x \in \delta \Omega$ as well. Hence, since

$$
\left|\mathbb{L}_{1} u_{1}-\mathbb{L}_{1} u_{1}^{\prime}\right|_{0} \geq\left|u_{1}\left(x_{1}, t_{1}\right)-\mathbb{L}_{1} u_{1}^{\prime}\left(x_{1}, t_{1}\right)\right|,
$$

$\mathbb{L}_{1}^{-1}$ exists on $R\left(\mathbb{L}_{1}\right)$ and satisfies

$$
\left|\mathbb{L}_{1}^{-1} u_{1}-\mathbb{L}_{1}^{-1} u_{1}^{\prime}\right|_{0} \leq \gamma_{1}^{-1}\left|u_{1}-u_{1}^{\prime}\right|_{0}
$$


II. We prove next that $\mathbb{L}_{2}^{-1}$ exists and there is a $\gamma_{2}>0$ such that

$$
\left|\mathbb{L}_{2} u_{2}-\mathbb{L}_{2} u_{2}^{\prime}\right|_{0} \geq \gamma_{2}\left|u_{2}-u_{2}^{\prime}\right|_{0}
$$

for any two $u_{2}, u_{2}^{\prime} \in D\left(\mathbb{L}_{2}\right)$. We define $v \equiv u_{2}-u_{2}^{\prime}$, and let $\left(x_{2}, t_{2}\right)$ be a point in $\Omega \times[0, T]$ such that

$$
|v|_{0}=\left|v\left(x_{2}, t_{2}\right)\right| \text {. }
$$

It is important to note that $v(x, T)=0$ for any $x \in \Omega$, so that $t_{2}<T$.

Let us show that

$$
v\left(x_{2}, t_{2}\right)\left(\mathbb{L}_{2} u_{2}-\mathbb{L}_{2} u_{2}^{\prime}\right) \geq \gamma_{2}|v|_{0}^{2} .
$$

If $v\left(x_{2}, t_{2}\right)=0$ then the inequality above is trivially verified. If $v\left(x_{2}, t_{2}\right) \neq 0, v\left(x_{2}, t_{2}\right)$ is rather a positive maximum or a negative minimum in $\Omega \times[0, T)$. Hence,

$$
v_{t}\left(x_{2}, t_{2}\right)=0
$$

whenever $t_{2}<T$. As in point I, $x_{2}$ can lie either in $\stackrel{\Omega}{\Omega}$ or in $\delta \Omega$ :

II.1 If $x_{2} \in \stackrel{\Omega}{\Omega}$ then

$$
v_{x}\left(x_{2}, t_{2}\right)=0
$$

and $v\left(x_{2}, t_{2}\right) v_{x x}\left(x_{2}, t_{2}\right) \leq 0$. We can then write:

$$
\begin{gathered}
-v\left(x_{2}, t_{2}\right)\left[v_{t}\left(x_{2}, t_{2}\right)+v_{x x}\left(x_{2}, t_{2}\right)\right] \geq 0, \\
-v\left(x_{2}, t_{2}\right)\left[\mathbb{L}_{2} u_{2}-\mathbb{L}_{2} u_{2}^{\prime}+\bar{c}_{2}\left(u_{2}-u_{2}^{\prime}\right)\right] \geq 0, \\
-v\left(x_{2}, t_{2}\right)\left[\mathbb{L}_{2} u_{2}-\mathbb{L}_{2} u_{2}^{\prime}\right] \geq \bar{c}_{2} v\left(x_{2}, t_{2}\right)\left(u_{2}-u_{2}^{\prime}\right) \geq \gamma_{2}|v|_{0}^{2},
\end{gathered}
$$

for any $0<\gamma_{2} \leq \bar{c}_{2}$. On the other hand,

$$
|-v|_{0}\left|\mathbb{L}_{2} u_{2}-\mathbb{L}_{2} u_{2}^{\prime}\right|_{0} \geq-v\left(x_{2}, t_{2}\right)\left(\mathbb{L}_{2} u_{2}-\mathbb{L}_{2} u_{2}^{\prime}\right)
$$

and $|-v|_{0}=|v|_{0}$. Therefore,

$$
\left|\mathbb{L}_{2} u_{2}-\mathbb{L}_{2} u_{2}^{\prime}\right|_{0} \geq \gamma_{2}\left|u_{2}-u_{2}^{\prime}\right|_{0}
$$

Again, as in Pao (1992), this implies that $\mathbb{L}_{2}^{-1}$ exists and satisfies

$$
\left|\mathbb{L}_{2}^{-1} u_{2}-\mathbb{L}_{2}^{-1} u_{2}^{\prime}\right|_{0} \leq \gamma_{2}^{-1}\left|u_{2}-u_{2}^{\prime}\right|_{0}
$$


II.2 If $x_{2} \in \delta \Omega$ then, since $\lim _{x \rightarrow \delta \Omega} v_{t}(x, t)=0$ for all $t$, there exists $\left(x_{\epsilon}, t_{\epsilon}\right) \in \stackrel{\Omega}{ } \times[0, T)$ and $\epsilon$ sufficiently small such that

$$
\left\{\begin{array}{l}
v\left(x_{2}, t_{2}\right) v_{t}\left(x_{\epsilon}, t_{\epsilon}\right) \leq \frac{\epsilon}{2}|v|_{0}^{2}, \\
v\left(x_{2}, t_{2}\right) v_{x x}\left(x_{\epsilon}, t_{\epsilon}\right) \leq \frac{\epsilon}{2}|v|_{0}^{2} .
\end{array}\right.
$$

In particular, we will restrict $\epsilon$ to $\epsilon \leq \bar{c}_{2}\left(x_{2}, t_{2}\right)$. Therefore,

$$
-v\left(x_{2}, t_{2}\right)\left[v_{t}\left(x_{\epsilon}, t_{\epsilon}\right)+v_{x x}\left(x_{\epsilon}, t_{\epsilon}\right)\right] \geq-\epsilon|v|_{0}^{2},
$$

that is,

$$
-\left.v\left(x_{2}, t_{2}\right)\left[\mathbb{L}_{2} u_{2}-\mathbb{L}_{2} u_{2}^{\prime}+\bar{c}_{2}\left(u_{2}-u_{2}^{\prime}\right)\right]\right|_{\left(x_{\epsilon}, t_{\epsilon}\right)} \geq-\epsilon|v|_{0}^{2} .
$$

It is also true that

$$
\left|v\left(x_{2}, t_{2}\right)\right|_{0}\left|\mathbb{L}_{2} u_{2}-\mathbb{L}_{2} u_{2}^{\prime}\right|_{0} \geq-\left.v\left(x_{2}, t_{2}\right)\left[\mathbb{L}_{2} u_{2}-\mathbb{L}_{2} u_{2}^{\prime}\right]\right|_{\left(x_{\epsilon}, t_{\epsilon}\right)} .
$$

Using (31) and (32) we obtain

$$
\left|v\left(x_{2}, t_{2}\right)\right|_{0}\left|\mathbb{L}_{2} u_{2}-\mathbb{L}_{2} u_{2}^{\prime}\right|_{0} \geq-\epsilon|v|_{0}^{2}+\left.\bar{c}_{2}\left(x_{\epsilon}, t_{\epsilon}\right)\left|v\left(x_{2}, t_{2}\right)\right|_{0}\left(u_{2}-u_{2}^{\prime}\right)\right|_{\left(x_{\epsilon}, t_{\epsilon}\right)} .
$$

Therefore,

$$
\left|\mathbb{L}_{2} u_{2}-\mathbb{L}_{2} u_{2}^{\prime}\right|_{0} \geq-\epsilon|v|_{0}+\left.\bar{c}_{2}\left(x_{\epsilon}, t_{\epsilon}\right)\left(u_{2}-u_{2}^{\prime}\right)\right|_{\left(x_{\epsilon}, t_{\epsilon}\right)} .
$$

Besides, by continuity and since $u_{2, x}(x, t)=0$ for any point in $\delta \Omega$, we also know that

$$
\lim _{\left(x_{\epsilon}, t_{\epsilon}\right) \rightarrow\left(x_{2}, t_{2}\right)}\left(u_{2}-u_{2}^{\prime}\right)\left(x_{\epsilon}, t_{\epsilon}\right)=v\left(x_{2}, t_{2}\right) .
$$

We can choose any $0<\gamma_{2} \leq \bar{c}_{2}\left(x_{\epsilon}, t_{\epsilon}\right)-\epsilon$, and take the limit when $\left(x_{\epsilon}, t_{\epsilon}\right) \rightarrow$ $\left(x_{2}, t_{2}\right)$. Consequently,

$$
\begin{gathered}
-\epsilon|v|_{0}+\left.\bar{c}_{2}\left(x_{\epsilon}, t_{\epsilon}\right)\left(u_{2}-u_{2}^{\prime}\right)\right|_{\left(x_{\epsilon}, t_{\epsilon}\right)} \rightarrow-\epsilon|v|_{0}+\left.\bar{c}_{2}\left(x_{2}, t_{2}\right)\left(u_{2}-u_{2}^{\prime}\right)\right|_{\left(x_{2}, t_{2}\right)} \\
=-\epsilon|v|_{0}+\bar{c}_{2}\left(x_{2}, t_{2}\right)|v|_{0}=\left[-\epsilon+\bar{c}_{2}\left(x_{2}, t_{2}\right)\right]|v|_{0} .
\end{gathered}
$$

Hence, (33) implies that

$$
\left|\mathbb{L}_{2} u_{2}-\mathbb{L}_{2} u_{2}^{\prime}\right|_{0} \geq\left[-\epsilon+\bar{c}_{2}\left(x_{2}, t_{2}\right)\right]|v|_{0} \geq \gamma_{2}|v|_{0}
$$

As a direct consequence of Lemma B1, we can state the following result: 
Corollary B1. Taking $\gamma=\min \left\{\gamma_{1}, \gamma_{2}\right\}$, the operator $\mathbb{L} \equiv\left(\mathbb{L}_{1}, \mathbb{L}_{2}\right)^{\prime}$ verifies

$$
\left\|\mathbb{L}^{-1} u-\mathbb{L}^{-1} u^{\prime}\right\|_{0} \leq \gamma^{-1}\left\|u-u^{\prime}\right\|_{0}
$$

for $u, u^{\prime} \in R(\mathbb{L})$.

Under the assumptions of the theorem, there exist functions $K_{i}=K_{i}(x, t)$ in $\Omega \times$ $[0, T]$ with values in $\mathbb{R}$ such that, for any $u, v$ in the domain of $f_{i}$,

$$
\left|F_{i}\left(u_{1}, u_{2}\right)-F_{i}\left(v_{1}, v_{2}\right)\right| \leq K_{i}|u-v|
$$

We define $K \equiv K_{1}+K_{2}$. Let $\bar{K}$ be an upper bound for $K$. As we will see in the next lemma, one can then conclude that $\mathbb{L}^{-1} F(u)$ is a contraction. The limits of our sequences therefore coincide and define the unique solution of $(I)$ :

Lemma B2. Let $\gamma>\bar{K}$, and consider $F(u)$ defined in (9) and $\mathbb{L}^{-1} F$ a mapping from $X \subset C(\Omega \times[0, T])$ into $C(\Omega \times[0, T])$. Then, $\mathbb{L}^{-1} F$ possesses the contraction property

$$
\left\|\mathbb{L}^{-1} F(u)-\mathbb{L}^{-1} F\left(u^{\prime}\right)\right\|_{0} \leq \frac{\bar{K}}{\gamma}\left\|u-u^{\prime}\right\|_{0}
$$

for all $u, u^{\prime} \in X$.

Proof. For each $i=1,2$ and $u \in X \subset C(\Omega \times[0, T])$, the function $F_{i}(u)$ is in X. This implies that $F_{i}(u) \in R\left(\mathbb{L}_{i}\right)$, thus $F(u) \in R(\mathbb{L})$. Hence, by Lemma $1, \mathbb{L}^{-1}$ is well defined on $X$. Since $F_{i}$ verifies

$$
\left|F_{i}(u)-F_{i}\left(u^{\prime}\right)\right| \leq K_{i}\left|u-u^{\prime}\right| \leq K_{i}\left|u-u^{\prime}\right|_{0}
$$

function $F$ satisfies the condition

$$
\left\|F(u)-F\left(u^{\prime}\right)\right\|_{0} \leq \bar{K}\left\|u-u^{\prime}\right\|_{0}
$$

for all $u, u^{\prime} \in X$. We also know from Corollary B1 that

$$
\left\|\mathbb{L}^{-1} F(u)-\mathbb{L}^{-1} F\left(u^{\prime}\right)\right\|_{0} \leq \gamma^{-1}\left\|F(u)-F\left(u^{\prime}\right)\right\|_{0}
$$

Taking (34) and (35) together, we obtain

$$
\left\|\mathbb{L}^{-1} F(u)-\mathbb{L}^{-1} F\left(u^{\prime}\right)\right\|_{0} \leq \gamma^{-1} \bar{K}\left\|u-u^{\prime}\right\|_{0}
$$

which proves the lemma. 


\section{Theorem 2 proof}

We prove this result by contradiction. Let $u \in C^{2,1}(\Omega \times[0, T])$ be a solution to $(I)$ and assume that $u \nsubseteq<\tilde{u}, \hat{u}>$. We may encounter six different cases:

i) $u$ equals the lower bound of $\langle\tilde{u}, \hat{u}\rangle$ in at least one point $\left(x_{0}, t_{0}\right)$, that is to say, $u\left(x_{0}, t_{0}\right)=\tilde{u}\left(x_{0}, t_{0}\right)$ and $\tilde{u}(x, t)<u(x, t)$ for all other $(x, t) \in \Omega \times[0, T] \backslash\left(x_{0}, t_{0}\right)$.

ii) $u$ equals the upper bound of $\left\langle\tilde{u}, \hat{u}>\right.$ in at least one point $\left(x_{0}, t_{0}\right)$, that is to say $u\left(x_{0}, t_{0}\right)=\hat{u}\left(x_{0}, t_{0}\right)$ and $u(x, t)<\hat{u}(x, t)$ for all other $(x, t) \in \Omega \times[0, T] \backslash\left(x_{0}, t_{0}\right)$.

iii) $u$ is smaller than $\left(\tilde{u}_{1}, \tilde{u}_{2}\right)$ in at least one point.

$i v) u$ is larger than $\left(\hat{u}_{1}, \hat{u}_{2}\right)$ in at least one point.

$v) u$ is smaller than $\left(\tilde{u}_{1}, \tilde{u}_{2}\right)$ in all points.

vi) $u$ is larger than $\left(\hat{u}_{1}, \hat{u}_{2}\right)$ in all points.

We shall focus on points $i$ ), iii) and $v$ ). Similar reasoning can be applied to study the remaining cases.

i) There exists a point $\left(x_{0}, t_{0}\right)$ at which $\tilde{u}\left(x_{0}, t_{0}\right)=u\left(x_{0}, t_{0}\right)$ and $\tilde{u}(x, t)<u(x, t)$ for all other $(x, t) \in \Omega \times[0, T] \backslash\left(x_{0}, t_{0}\right)$. We define $v(x, t) \equiv u(x, t)-\tilde{u}(x, t)$, then $\left(x_{0}, t_{0}\right)$ is a minimum for $v$. Therefore

$$
\begin{aligned}
& v_{t}\left(x_{0}, t_{0}\right)=0, \\
& v_{x x}\left(x_{0}, t_{0}\right)>0 .
\end{aligned}
$$

Consequently, for $v=\left(v_{1}, v_{2}\right)$, we have that $\left.\mathbb{L}_{1} v_{1}\right|_{\left(x_{0}, t_{0}\right)}<0$ and $\left.\mathbb{L}_{2} v_{2}\right|_{\left(x_{0}, t_{0}\right)}>0$. However,

$$
\mathbb{L}_{1} v_{1}=\mathbb{L}_{1}\left(u_{1}-\tilde{u}_{1}\right)=\mathbb{L}_{1} u_{1}-\mathbb{L}_{1} \tilde{u}_{1}=f_{1}\left(u_{1}, u_{2}\right)-f_{1}\left(\tilde{u}_{1}, \tilde{u}_{2}\right)+\underline{a}_{1}\left(u_{1}-\tilde{u}_{1}\right) \geq 0 .
$$

The expression above is positive under the assumptions because, as we have seen in the proof of Proposition B1, either $f_{1}$ is increasing in both arguments, with $\underline{a}_{1} \geq 0$ (which yields the result directly), or because $\underline{a}_{2} \leq 0$. Notice that in the later case

$$
\mathbb{L}_{1} v_{1}(x, t) \geq-\underline{a}_{2}\left(u_{2}-\tilde{u}_{2}\right) \geq 0 .
$$

Hence, we achieve a contradiction. 
Similarly, one can verify that

$$
\mathbb{L}_{2} v_{2}=\mathbb{L}_{2}\left(u_{2}-\tilde{u}_{2}\right)=\mathbb{L}_{2} u_{2}-\mathbb{L}_{2} \tilde{u}_{2}=f_{2}\left(u_{1}, u_{2}\right)-f_{2}\left(\tilde{u}_{1}, \tilde{u}_{2}\right)-\bar{c}_{2}\left(u_{2}-\tilde{u}_{2}\right) \leq 0,
$$

which is negative under the theorem assumptions. As before, this result is directly confirmed if $f_{2}$ is decreasing in both arguments, with $\bar{c}_{2} \geq 0$. Otherwise, we can show that, for $\bar{c}_{1} \leq 0$ :

$$
\mathbb{L}_{2} v_{2} \leq \bar{c}_{1}\left(u_{1}-\tilde{u}_{1}\right) \leq 0 \text {. }
$$

A contradiction arises again. Then, we finally conclude that $u$ and $\tilde{u}$ cannot coincide in one point.

iii) In the case when $u$ is smaller than the lower bound of $<\tilde{u}, \hat{u}>$ in at least one point, by continuity, there must be at least a point $\left(x_{0}, t_{0}\right)$ at which $u\left(x_{0}, t_{0}\right)=\tilde{u}\left(x_{0}, t_{0}\right)$. But we just showed in point $i$ ) that this is impossible.

$v$ ) Notice that $u, \tilde{u}$ are continuous functions on a compact space. The function $v$, defined as $v \equiv \tilde{u}-u$, achieves then a minimum at a point denoted by $\left(x_{0}, t_{0}\right)$. Therefore,

$$
\begin{aligned}
& v_{t}\left(x_{0}, t_{0}\right)=0, \\
& v_{x x}\left(x_{0}, t_{0}\right)>0 .
\end{aligned}
$$

Hence, $\left.\mathbb{L}_{1} v_{1}\right|_{\left(x_{0}, t_{0}\right)}<0$ and $\left.\mathbb{L}_{2} v_{2}\right|_{\left(x_{0}, t_{0}\right)}>0$. However, as in case $\left.i\right)$ above,

$$
\mathbb{L}_{1} v_{1}=\mathbb{L}_{1}\left(\tilde{u}_{1}-u_{1}\right)=\mathbb{L}_{1} \tilde{u}_{1}-\mathbb{L}_{1} u_{1}=f_{1}\left(\tilde{u}_{1}, \tilde{u}_{2}\right)-f_{1}\left(u_{1}, u_{2}\right)+\underline{a}_{1}\left(u_{1}-\tilde{u}_{1}\right) \geq 0,
$$

which leads to a contradiction under the theorem assumptions. Similarly,

$$
\mathbb{L}_{2} v_{2}=\mathbb{L}_{2}\left(\tilde{u}_{2}-u_{2}\right)=\mathbb{L}_{2} \tilde{u}_{2}-\mathbb{L}_{2} u_{2}=f_{2}\left(\tilde{u}_{1}, \tilde{u}_{2}\right)-f_{2}\left(u_{1}, u_{2}\right)-\bar{c}_{2}\left(u_{2}-\tilde{u}_{2}\right) \leq 0 .
$$

The contradiction arises because $\left.\mathbb{L}_{2} v_{2}\right|_{\left(x_{0}, t_{0}\right)}>0$. This finishes the proof.

\section{A numerical algorithm}

In this appendix we develop an algorithm to numerically compute the optimal solution to our optimal control problem. Since the algorithm is based on the iterative method presented in Theorem 1, it will require a lower or an upper solution to start iterating. For illustration purposes let us build a sequence $\left\{u_{1}^{k}, u_{2}^{k}\right\}_{k \in \mathbb{N}}$ with $\left(u_{1}^{0}, u_{2}^{0}\right)=\left(\tilde{u}_{1}, \tilde{u}_{2}\right)$. The algorithm can be summarized in the following 3 steps: 
Step 1: Set a stopping criterion $\Delta$ and build a grid to represent the space-time set $\Omega \times[0, T], \Omega \simeq\{D x, 2 * D x, \ldots, N * D x\}$ and $[0, T] \simeq\{D t, 2 * D t, \ldots, J * D t\}$. We initialize the series $\left\{u_{1}^{k} u_{2}^{k}\right\}_{k \in \mathbb{N}}$ as

$$
\begin{aligned}
& \left(\underline{u}_{1}^{0}, \underline{u}_{2}^{0}\right)=\left(\tilde{u}_{1}, \tilde{u}_{2}\right), \\
& \left(\underline{w}_{1}^{0}, \underline{w}_{2}^{0}\right)=\left(\tilde{w}_{1}, \tilde{w}_{2}\right) .
\end{aligned}
$$

Step 2: For every $k$ and given $\left(u_{1}^{k-1}, u_{2}^{k-1}\right)$ and $\left(w_{1}^{k-1}, w_{2}^{k-1}\right)(x, t)=\left(u_{1}^{k-1}, u_{2}^{k-1}\right)(x, T-$ $t)$, compute

$$
\left\{\begin{array}{l}
\mathbb{L}_{1} u_{1}^{k}=F_{1}\left(u_{1}^{k-1}, u_{2}^{k-1}\right) \\
u_{1}^{k}(x, 0)=\frac{\zeta}{k} \tilde{u}_{1}(x, 0)+\left(1-\frac{\zeta}{k}\right) u_{1}(x, 0), \\
\lim _{x \rightarrow \delta \Omega} u_{1, x}^{k}=0
\end{array}\right.
$$

with $0<\zeta<1$, and

$$
\left\{\begin{array}{l}
\mathbb{L}_{2}^{\prime} w_{2}^{k}=-F_{2}\left(w_{1}^{k-1}, w_{2}^{k-1}\right), \\
w_{2}^{k}(x, 0)=\frac{\phi}{k} \tilde{w}_{2}(x, 0)+\left(1-\frac{\phi}{k}\right) \Psi_{1}^{\prime}\left(w_{1}^{k-1}(x, 0), x\right), \\
\lim _{x \rightarrow \delta \Omega} w_{2, x}^{k}=0,
\end{array}\right.
$$

with $0<\psi<1$.

Step 3: After every $k$, we compute the distance between $\left(u_{1}^{k-1}, u_{2}^{k-1}\right)$ and $\left(u_{1}^{k}, u_{2}^{k}\right)$. If the distance is smaller than $\Delta$, then STOP. If not, set $k=k+1$ and go to step 2 .

\section{References}

Acemoglu D. (2009), Introduction to Modern Economic Growth, Princeton University Press, New Jersey.

Ahmed N. (1977), "Optimal Control of a Class of Strongly Nonlinear Parabolic Systems", Journal of Mathematical Analysis and Applications, 61, 188-207.

Attanasio O. and M. Browning (1995), "Consumption over the Life Cycle and over the Business Cycle", American Economic Review, 85(5), 1118-1137.

Attanasio O. and G. Weber (1993), "Consumption, the Interest Rate and Aggregation", Review of Economic Studies, 60(3), 631-649. 
Baldwin R. and P. Martin (2004), "Agglomeration and regional growth", in Handbook of Regional and Urban Economics, vol.4, V. Henderson and J. Thisse (eds.), NorthHolland, Amsterdam, The Netherlands.

Baldwin R. and P. Krugman (2004), "Agglomeration, Integration and Tax Harmonisation", European Economic Review, 48(1), 1-23.

Barbu V. and T. Precupanu (2012), Convexity and optimization in Banach spaces, Springer Science \& Business Media, New York City.

Barro R., and X. Sala-i-Martin (2004), Economic Growth, 2nd Edition, The MIT Press, Cambridge, Massachusetts.

Barro R., G. Mankiw, and X. Sala-i-Martin (1995), "Capital Mobility in Neoclassical Models of Growth", American Economic Review, 85(1), 103-115.

Bebernes J. and K. Schmitt, K (1979), "On the existence of maximal and minimal solutions for parabolic partial differential equations", Proceedings of the American Mathematical Society, 73(2), 211-218.

Bental B. (1985), "Is Capital Mobility Always Desirable? A Welfare Analysis of Portfolio Autarky in a Growing Economy", International Economic Review, 26(1), 203-212.

Blanchet A., P. Mossay, and P. Santambrogio (2016), "Existence and uniqueness of equilibrium for a spatial model of social interactions", International Economic Review, $57,31-59$.

Boucekkine R., C. Camacho, and B. Zou (2009), "Bridging the gap between growth theory and the new economic geography: The spatial Ramsey model", Macroeconomic Dynamics, 13, 20-45.

Boucekkine R., C. Camacho, and G. Fabbri (2013a), "Spatial dynamics and convergence: The spatial AK model", Journal of Economic Theory, 148, 2719-2736.

Boucekkine R., C. Camacho, and G. Fabbri (2013b), "On the optimal control of some parabolic partial differential equations arising in economics", Serdica Mathematical Journal, 39, 1001-1024.

Brito P. (2004), "The dynamics of growth and distribution in a spatially heterogenous world", WP 13/2004, ISEG Working Papers. 
Brock W. and A. Xepapadeas (2008), "Diffusion-induced instability and pattern formation in infinite horizon recursive optimal control", Journal of Economic Dynamics and Control, 32, 2745-2787.

Camacho C. and A. Perez-Barahona (2015), "Land use dynamics and the environment", Journal of Economic Dynamics and Control, 52, 96-118.

Camacho C., Zou B. and Briani M. (2008), "On the dynamics of capital accumulation across space", European Journal of Operational Research, 186(2), 451-465.

Chandra J., V. Lakshmikantham and S. Leela (1978), "A monotone method for infinite systems of nonlinear boundary value problems", Archive for Rational Mechanics and Analysis, 68(2), 179-190.

Chetty R. (2006), "A New Method of Estimating Risk Aversion", American Economic Review, 96(5), 1821-1834.

Costello C. and S. Polasky (2008), "Optimal harvesting of stochastic spatial resources" Journal of Environmental Economics and Management, 56, 1-18.

Desmet K. and E. Rossi-Hansberg (2009), "Spatial Growth and Industry Age", Journal of Economic Theory, 144(6), 2477-2502.

Desmet K. and E. Rossi-Hansberg (2010), "On spatial dynamics", Journal of Regional Science, 50(1), 43-63.

Desmet K. and E. Rossi-Hansberg (2014), "Spatial development", American Economic Review, 104(4), 1211-1243.

Desmet K. and E. Rossi-Hansberg (2015), "On the spatial economic impact of global warming", Journal of Urban Economics, 88, 16-37.

Ekeland I. (1974), "On the variational principle", Journal of Mathematical Analysis and Applications, 47, 324-353.

European Union (2008), "Consolidated version of the Treaty on the Functioning of the European Union. Part 3: Union policies and internal actions. Title IV: Free movement of persons, services and capital. Chapter 4: Capital and payments. Article 63 (ex Article 56 TEC)", The Official Journal of the European Union 115, 71-71.

Fabbri G. (2016), "Geographical structure and convergence: A note on geometry in spatial growth models", Journal of Economic Theory, 162, 114-136. 
Fattorini H., (1990), "Some remarks on Pontryagin's maximum principle for infinite dimensional control problemsf", in Perspectives in Control Theory, Springer Science+Business Media, 12-25.

Friedman A. (1959), "Convergence of solutions of parabolic equations to a steady state", Journal of Mathematics and Mechanics , 8, 57-76.

Galor O. (1996), "Convergence? Inferences from theoretical models", The Economic Journal, 106, 1056-1069.

Hadamard J., (1923), Lectureson the Cauchy Problem in Linear Partial Differential Equations. Yale University Press.

Kehoe T., D. Levine, and P. Romer (1990), "Determinacy of Equilibria in Dynamic Models with Finitely Many Consumers", Journal of Economic Theory, 50 (1), 1-21.

Kose A., E. Prasad, K. Rogoff, and S. Wei (2010), "Financial Globalization and Economic Policies", in Handbook of Development Economics, vol.5, D. Rodrik and M. Rosenzweig (eds.), North-Holland, Amsterdam, The Netherlands.

Lions J. (1966a), "Optimisation pour certaines classes d'équations d'évolution non linéaires", Annali di Matematica Pura ed Applicata, 72(1), 275-294.

Lions J. (1966b), "Sur quelques problèmes d'optimisation dans les equations d'evolution linéaires de type parabolique", in Applications of functional Analysis to Optimization, Caianiello (ed.), Academic Press, New York.

Lions J. (1972), "Some aspects of the optimal control of distributed parameter systems", Regional Conference Series in Applied Mathematics, SIAM, Philadenphia, PA.

Mossay P. and P. Picard (2011), "On spatial equilibria in a social interaction model", Journal of Economic Theory, 146, 2455-2477.

Pao C. (1992), Nonlinear Parabolic and Elliptic Equations, Plenum Press, New York.

Patel S. (1964), "The Economic Distance Between Nations: Its Origin, Measurement and Outlook", The Economic Journal, 74(293), 119-131.

Quah D. (1996a), "Convergence Empirics Across Economies with (Some) Capital Mobility", Journal of Economic Growth, 1, 95-124. 
Quah D. (1996b), "Twin peaks: growth and convergence in models of distribution dynamics", The Economic Journal, 106, 1045-1055.

Raymond J. and H. Zidani (1999), "Pontryagin's principle for Time-Optimal Problems", Journal of Optimization Theory and Applications, 101(2), 375-402.

Rodrik D. and T. van Ypersele (2001), "Captial mobility, distributive conflict and international tax coordination", Journal of International Economics, 54, 57-73.

Sattinger D. (1972), "Monotone Methods in Nonlinear Elliptic and Parabolic Boundary Value Problems", Indiana University Mathematics Journal, 21(11), 979-1000.

Thisse J. (2010), "Towards a unified theory of economic geography and urban economics", Journal of Regional Science, 50(1), 281-296. 